ARTICLE

Translational Therapeutics

\title{
Low-dose anti-inflammatory combinatorial therapy reduced cancer stem cell formation in patient-derived preclinical models for tumour relapse prevention
}

Bee Luan Khoo ${ }^{1}$, Gianluca Grenci, ${ }^{2,3}$, Joey Sze Yun Lim ${ }^{1}$, Yan Ping Lim ${ }^{4}$, July Fong ${ }^{5}$, Wei Hseun Yeap ${ }^{6}$, Su Bin Lim ${ }^{3,7}$, Song Lin Chua ${ }^{8}$, Siew Cheng Wong ${ }^{6}$, Yoon-Sim Yap ${ }^{9}$, Soo Chin Lee ${ }^{10,11}$, Chwee Teck Lim ${ }^{1,2,3,12}$ and Jongyoon Han ${ }^{1,13}$

BACKGROUND: Emergence of drug-resistant cancer phenotypes is a challenge for anti-cancer therapy. Cancer stem cells are identified as one of the ways by which chemoresistance develops.

METHOD: We investigated the anti-inflammatory combinatorial treatment (DA) of doxorubicin and aspirin using a preclinical microfluidic model on cancer cell lines and patient-derived circulating tumour cell clusters. The model had been previously demonstrated to predict patient overall prognosis.

RESULTS: We demonstrated that low-dose aspirin with a sub-optimal dose of doxorubicin for $72 \mathrm{~h}$ could generate higher killing efficacy and enhanced apoptosis. Seven days of DA treatment significantly reduced the proportion of cancer stem cells and colonyforming ability. DA treatment delayed the inhibition of interleukin- 6 secretion, which is mediated by both COX-dependent and independent pathways. The response of patients varied due to clinical heterogeneity, with $62.5 \%$ and $64.7 \%$ of samples demonstrating higher killing efficacy or reduction in cancer stem cell (CSC) proportions after DA treatment, respectively. These results highlight the importance of using patient-derived models for drug discovery.

CONCLUSIONS: This preclinical proof of concept seeks to reduce the onset of CSCs generated post treatment by stressful stimuli. Our study will promote a better understanding of anti-inflammatory treatments for cancer and reduce the risk of relapse in patients.

British Journal of Cancer (2019) 120:407-423; https://doi.org/10.1038/s41416-018-0301-9

\section{INTRODUCTION}

In the recent decade, there has been an increasing number of anti-cancer drug clinical trials. ${ }^{1}$ However, the efficacy of several drugs may be limited by the requirement for higher dosage in vivo to overcome pharmacokinetics issues. ${ }^{2}$ Another key factor in the lack of therapeutic efficacy is the inability to eliminate cancer cells completely, a process hindered by the heterogeneity and plasticity of human biological systems. ${ }^{3,4}$ Notably, stressful stimuli post treatment are known to have either a prodeath or prosurvival role and could drive cancer cells to become more metastatic and drug-resistant. ${ }^{5}$

The reduction of cancer stem cells (CSCs) post treatment is important as the emergence of CSCs via epithelial-mesenchymal transition (EMT) is identified as one of the ways by which chemoresistance develops. ${ }^{6-8}$ Other ways involve transporter pumps, ${ }^{9}$ genetic alteration, ${ }^{10}$ or exosomes. ${ }^{11}$ Hence, CSCs as key targets for anti-cancer strategies. ${ }^{12}$ CSCs may be found circulating in the bloodstream ${ }^{13}$ upon extrusion by primary tumours. ${ }^{14}$ Heterogeneity and plasticity of CSCs hinder complete eradication, ${ }^{15}$ which account for metastasis ${ }^{16}$ at distant sites even after successful treatment. ${ }^{17}$

It was previously shown that cancer patients on a supplement of aspirin had reduced cancer risk and longer overall survival than those who were not. ${ }^{18,19}$ Aspirin is a nonsteroidal antiinflammatory drug most commonly used to treat inflammatory diseases. The association between chronic inflammation and cancer $^{20,21}$ suggests that aspirin can be effective against cancer. Indeed, anti-cancer effects of aspirin have been established in colorectal cancer, ${ }^{19,22,23}$ oesophageal cancer $^{24}$ gastric cancer, ${ }^{25}$ liver cancer, ${ }^{26}$ and pancreatic cancer. ${ }^{27}$

In this proof of concept study, a range of therapeutic drug concentrations for $0-500 \mathrm{mg} / \mathrm{ml}$ aspirin (A) and $0-1 \mu \mathrm{M}$

\footnotetext{
${ }^{1}$ BioSystems and Micromechanics IRG, Singapore-MIT Alliance for Research and Technology Centre, Singapore, Singapore; ${ }^{2}$ Mechanobiology Institute, National University of Singapore, Singapore, Singapore; ${ }^{3}$ Department of Biomedical Engineering, National University of Singapore, Singapore, Singapore; ${ }^{4}$ Department of Biochemistry, Yong Loo Lin School of Medicine, National University of Singapore, Singapore, Singapore; ${ }^{5}$ Singapore Centre for Environmental Life Sciences Engineering, Singapore, Singapore; ${ }^{6}$ Singapore Immunology Network, Agency for Science, Technology and Research, Singapore, Singapore; ${ }^{7}$ NUS Graduate School for Integrative Sciences \& Engineering (NGS), National University of Singapore, Singapore, Singapore; ${ }^{8}$ Lee Kong Chian School of Medicine, Nanyang Technological University, Singapore, Singapore; ${ }^{9}$ Department of Medical Oncology, National Cancer Centre Singapore, Singapore, Singapore; ${ }^{10}$ Department of Hematology-Oncology, National University Cancer Institute, National University Hospital, Singapore, Singapore; ${ }^{11}$ Cancer Science Institute of Singapore, National University of Singapore, Singapore, Singapore; ${ }^{12}$ Biomedical Institute for Global Health Research and Technology, National University of Singapore, Singapore, Singapore and ${ }^{13}$ Department of Electrical Engineering and Computer Science, Department of Biological Engineering, Massachusetts Institute of Technology, Cambridge, Massachusetts, USA

Correspondence: Bee Luan Khoo (beeluan@smart.mit.edu) or Jongyoon Han (jyhan@mit.edu)
}

Received: 12 March 2018 Revised: 10 September 2018 Accepted: 20 September 2018

Published online: 4 February 2019 
408

doxorubicin (D), a common anti-cancer drug for breast cancer, were screened with a microfluidic culture and drug-screening assay validated for primary cell cultures. ${ }^{28}$ We demonstrated that low doses of aspirin $(\leq 500 \mathrm{mg} / \mathrm{ml})$ in combination with suboptimal doses of doxorubicin, a chemotherapy drug, could heighten anti-cancer effect within a relatively short period of time $(72 \mathrm{~h})$, specifically in breast cancer cell lines and patientderived clinical models. Cells treated with doxorubicin alone demonstrated an increase in CSC proportion over time (7 days). Conversely, cells under combinatorial DA treatment generated a significantly lower proportion of CSCs, leading to reduced cancer cell cluster formation or spheroid growth.

Under combinatorial DA treatment, there was also a reduction of metastatic-like phenotype as compared with cells treated with doxorubicin alone. This was despite the increase of interleukin-6 (IL-6) and JAK1 expression levels, which was owing to the inhibition of IL- 6 by combinatorial DA treatment, leading to an overall reduction of $\mathrm{CSCs}^{29,30}$ Combinatorial treatment also reduced oxidative stress in the cells, as evident by Calcein AM expression, 3-(4,5-Dimethyl-2-thiazolyl)-2,5-diphenyl-2H-tetrazolium bromide (MTT) and peroxidase assays. The effects of combinatorial DA treatment were also mediated by cyclooxygenase (COX)-related pathways. Prior studies have demonstrated that COX-2/prostaglandin E2 (PGE2) pathways are potent inhibitors of EMT for epithelial cells, ${ }^{31}$ and the resultant COX-2-derived PGE2 and PGD2 are mediators of anti-EMT. ${ }^{32}$ COX-2 was also highly expressed in triple-negative breast cancer and is associated with poorer prognosis. ${ }^{33}$

We demonstrated that the reduction of CSCs under combinatorial DA treatment was reflected in both the cancer cell clusters and patient-derived circulating tumour cells (CTC) cluster models. The CTC clusters were obtained under culture with our microfluidics assay with minimal processing, which vastly promotes efficiency and allows samples to be analysed after 2 weeks. ${ }^{34}$ Specialised microwells within the assay recapitulate the tumour microenvironment through the proximity of cancer cells, and co-culture with patient's own immune cells under hypoxia. Doxorubicin has been reported in several cases as ineffective in the eradication of resistant CSCs. ${ }^{35,36}$ To the best of our knowledge, our study is the first to connect the anti-cancer effects of aspirin and anti-cancer drugs with the reduction of CSCs, in a patient-specific manner.

Our results suggest that the combinatorial use of aspirin and doxorubicin have the potential to prolong overall patient survival, paving the way for further studies on the co-treatment of other chemotherapy drugs with aspirin. Such studies also demonstrate the potential to reinvent a new role for aspirin, an affordable drug that can be accessed by many patients globally. Although the efficacy of combinatorial drug regime would be heterogeneous among patient populations, CTC cluster-forming assay would allow one to quickly evaluate this for optimal management of metastasis. These preclinical studies will potentially benefit patients by reducing the risk of cancer relapse after treatment and revolutionise personalised medicine through cheap and noninvasive screening methods.

\section{RESULTS}

Preclinical drug-screening using the high-throughput CTC cluster assay

We first utilised a cancer cell cluster assay optimised for cluster formation of clinical primary CTCs from blood biopsy ${ }^{37}$ as a relevant model in vitro for uniform cluster formation. The assay was designed to incorporate a layer of customised ellipsoidal microwells of $250 \mu \mathrm{m}$ by $150 \mu \mathrm{m}$ and $150 \mu \mathrm{m}$ depth, to promote the formation of single cancer cell clusters at low cell seeding concentration ( 0.25 million cells per channel). The assay was integrated with microfluidic components to enable high- throughput screening of drug combinations, as well as valves to control and compartmentalise media waste disposal (Fig. 1a, Figure S1 A, B). The design of the assay is flexible owing to the use of microfabrication, and assays can be incorporated into any number of channels, which corresponds to the range of drug concentrations screened. In this work, we utilised either the 8channel or 10-channel device for drug-screening tests where the drugs administered can vary up to 10 different concentrations ( $n=8$ or 10) (Figure S1C) either with one or two of the drug concentrations (Figure S1D, E) by pump infusion or manual addition (Figure S1F).

The cancer cell cluster formation process involved the seeding of cells into the microwells, followed by maintenance under a combination of conditions such as hypoxia to mimic the tumour microenvironmental niche (Fig. 1b). Resultant cancer cell clusters were three-dimensional, multilayered, and under low shear. ${ }^{37}$ Minimal pre-processing reduced the damage to target CTCs and allow increased efficiency of CTC cluster formation. ${ }^{28,34,38}$ Resultant clusters were heterogeneous and contain subpopulations of cancer stem cells, depending on sample parameters. The presence of CTCs, CSCs and other constituents was previously identified by immunostaining and/or fluorescence in situ hybridisation. ${ }^{38}$ Other constituents identified within the clusters include residual immune cells, some of which were also populations located in the tumour microenvironment niche. Despite the label-free and growth factor-free conditions, there was still an extent of selection in our assay with respect to the proliferative and cluster-forming CTC populations. Nonetheless, these populations are of utmost relevance to cancer progression, and hence reflect the potential of patient's response to therapy.

Consistent cancer cell clusters by size could be generated within the microwells (Figure S2A) with various cancer types such as colorectal and breast cancer cell lines. The range of cluster diameter for a single sample was consistent throughout the assay (SW480: $136.1 \pm 7.1 \mu \mathrm{m}$, MDA-MB-231: $142.2 \pm 4.6 \mu \mathrm{m}$; Figure S2B) but comparison of cluster sizes across cell lines of different EMT phenotypes demonstrated that epithelial-like cancer cell lines led to smaller cancer cell clusters, possibly due to closer cell-cell interactions (Figure $\mathrm{S} 2 \mathrm{C}$ ). ${ }^{39}$

To evaluate the drug response after combinatorial DA therapy, we first validated the cellular viability of monolayer cultures after respective drug exposures. For cellular viability, we validated the IC50 values of aspirin alone, doxorubicin alone or combinatorial DA therapy on MDA-MB-231 as 2D monolayers (seeding density $10 \mathrm{k}$ cells; Figure S3). We confirmed that the range of aspirin concentrations used was mostly above median lethal dosage (IC50 value $=412.5 \mathrm{mg} / \mathrm{ml})$, whereas concentrations for treatment with doxorubicin alone (IC50 value $=0.1 \mu \mathrm{M})$ and combinatorial DA treatment with doxorubicin concentration fixed at $0.5 \mu \mathrm{M}(0.5 \mathrm{D})$ were within $50 \%$ lethal dosage range. In fact, viability rates of monolayer cultures after combinatorial DA therapy were below that of cultures after single $0.5 \mathrm{D}$ treatment, suggesting a higher killing efficacy. Viability rates were determined by live and dead staining using Calcein AM and SYTOX, respectively. Using these optimal drug concentrations, we evaluated killing efficacy, metastatic potential and the mechanisms of treatment using our preclinical model on cancer cell lines and clinical patients (Fig. 1c).

Combinatorial anti-inflammatory therapy improved killing efficacy and apoptosis onset

We next tested the combinatorial DA therapy of doxorubicin and aspirin (DA) on three-dimensional (3D) cancer cell clusters. When cancer cell clusters were untreated over 7 days, viability rates of these cultures remained relatively constant $(p<0.01$; Figure S4). Vehicle samples were subjected to the same dimethyl sulfoxide (DMSO) concentration as those under respective drug treatments. After treatment with aspirin alone, viability rate of healthy blood cells remained constant after short-term exposure $(72 \mathrm{~h})$ to varied 
a
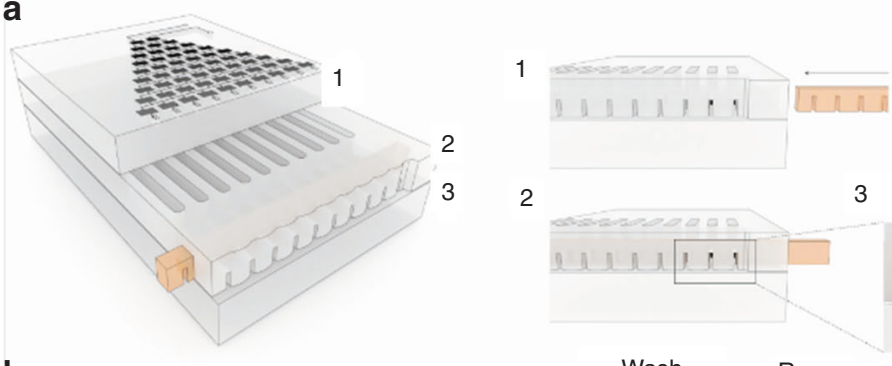

3 Close Open

b

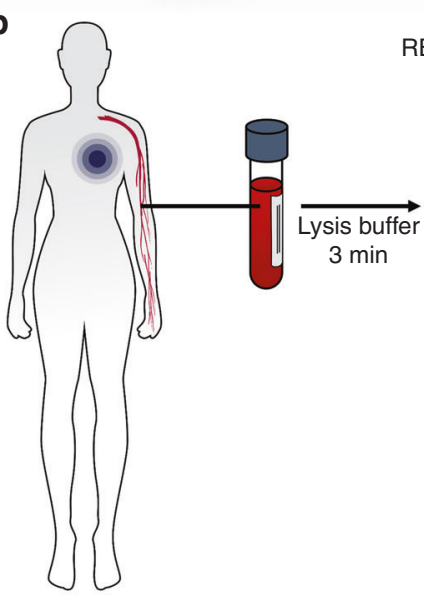

RBC lysis

Wash

Resuspend
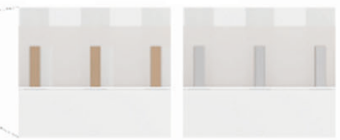

excess RBC

pellet

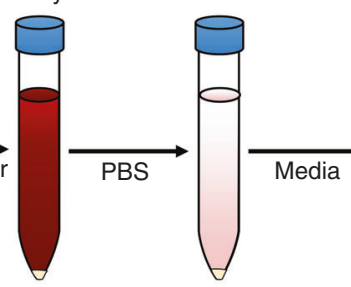

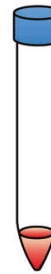

Seed cells

c

Combinational therapy

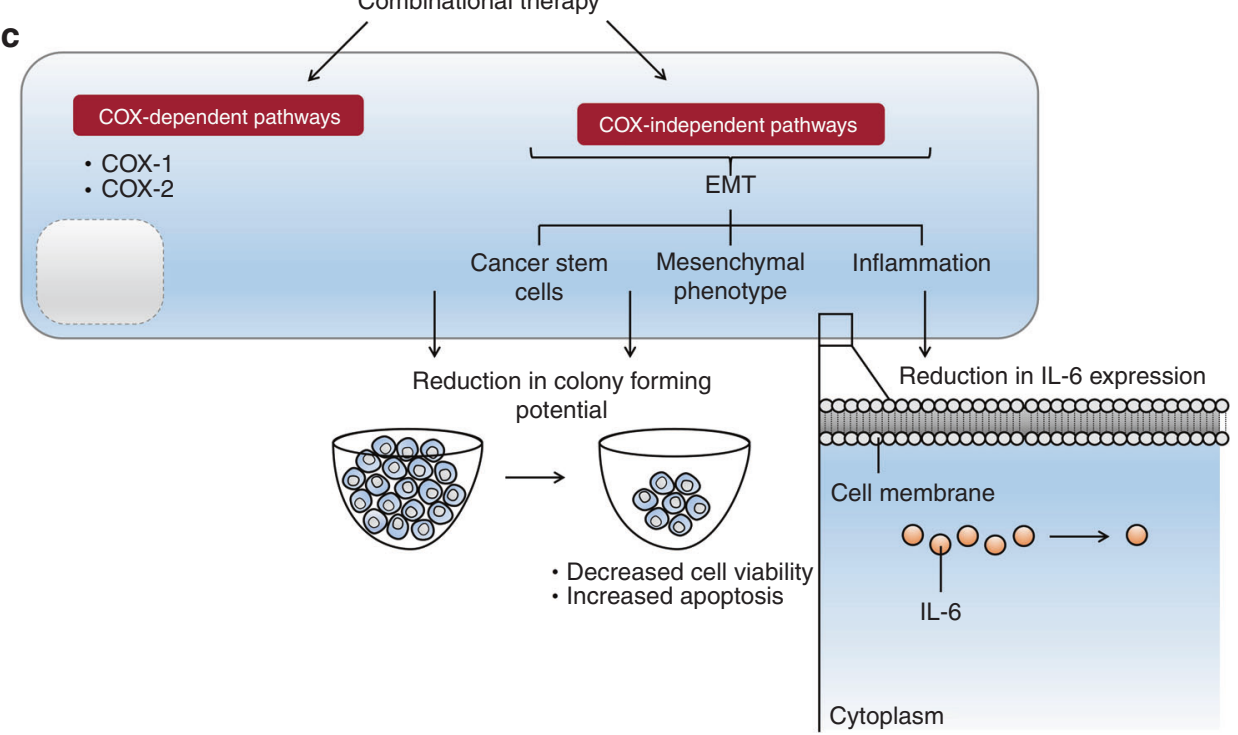

Fig. 1 Overview of assay design, screening, and therapeutic outcomes. a Schematics of the complete device (left panel), with details of valve design. In this portion of the device, the open channels were operated with an open/closed valve actuated by simply sliding in or out a bar with openings corresponding to the position of the channels. b Workflow for cancer cell cluster establishment and subsequent downstream analyses. A tumour-on-chip-like assay can be established under factors that aim to mimic the tumour microenvironmental niche, using a single pre-processing step to remove red blood cells prior to seeding. c The proposed mechanism of anti-inflammatory combinatorial treatment screened with the preclinical patient-derived model

dosages of aspirin $(0-500 \mathrm{mg} / \mathrm{ml})$ (Fig. 2a, b). After treatment with only $250 \mathrm{mg} / \mathrm{ml}$ aspirin concentration, the viability of the cultures remained constant over exposure periods of both $72 \mathrm{~h}$ and 7 days $(p<0.01$ ), relative to untreated samples (Fig. $2 c$, d) (Figure S5A). After treatment with doxorubicin alone, viability percentage dropped sharply beyond drug concentrations of $0.65 \mu \mathrm{M}(65 \%)$ for both cultures of healthy blood cells (Inhibitory concentration at $50 \%$ viability $(\mathrm{IC} 50)=0.75 \mu \mathrm{M})$ and cancer cell line $(\mathrm{IC50}=$ $0.84 \mu \mathrm{M})$ ) (Fig. 2e, f) (Figure S5B).
To investigate if the addition of aspirin would increase the anticancer effects of doxorubicin, we only used drug concentration levels that will yield minimal toxicity ( $>50 \%$ viability after $72 \mathrm{~h}$ treatment), hence only $0-50 \%(0-0.5 \mu \mathrm{M})$ of doxorubicin concentrations were used for subsequent combinatorial therapy experiments (Figure S6). For the treatment with $0.5 \mu \mathrm{M}$ doxorubicin concentration alone $(0.5 \mathrm{D})$, viability ratio could be reduced to $0.59 \pm 0.2$ after 7 days of exposure, relative to untreated samples (Fig. $2 \mathrm{~g}, \mathrm{~h}$ ), although the reduction was not significant. 

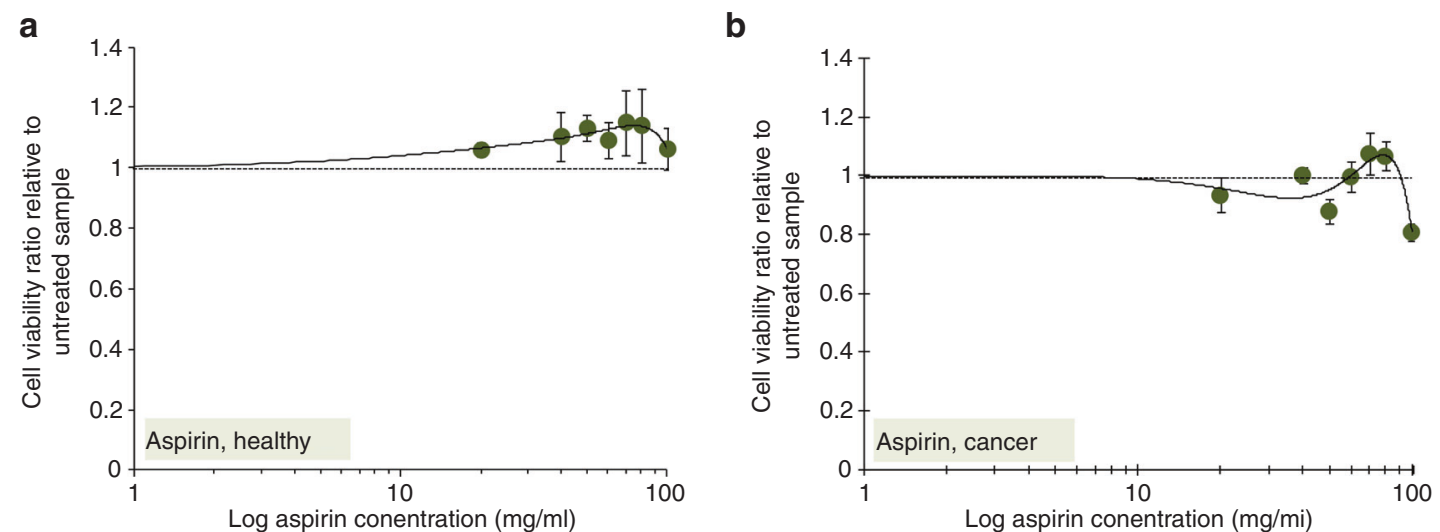

C
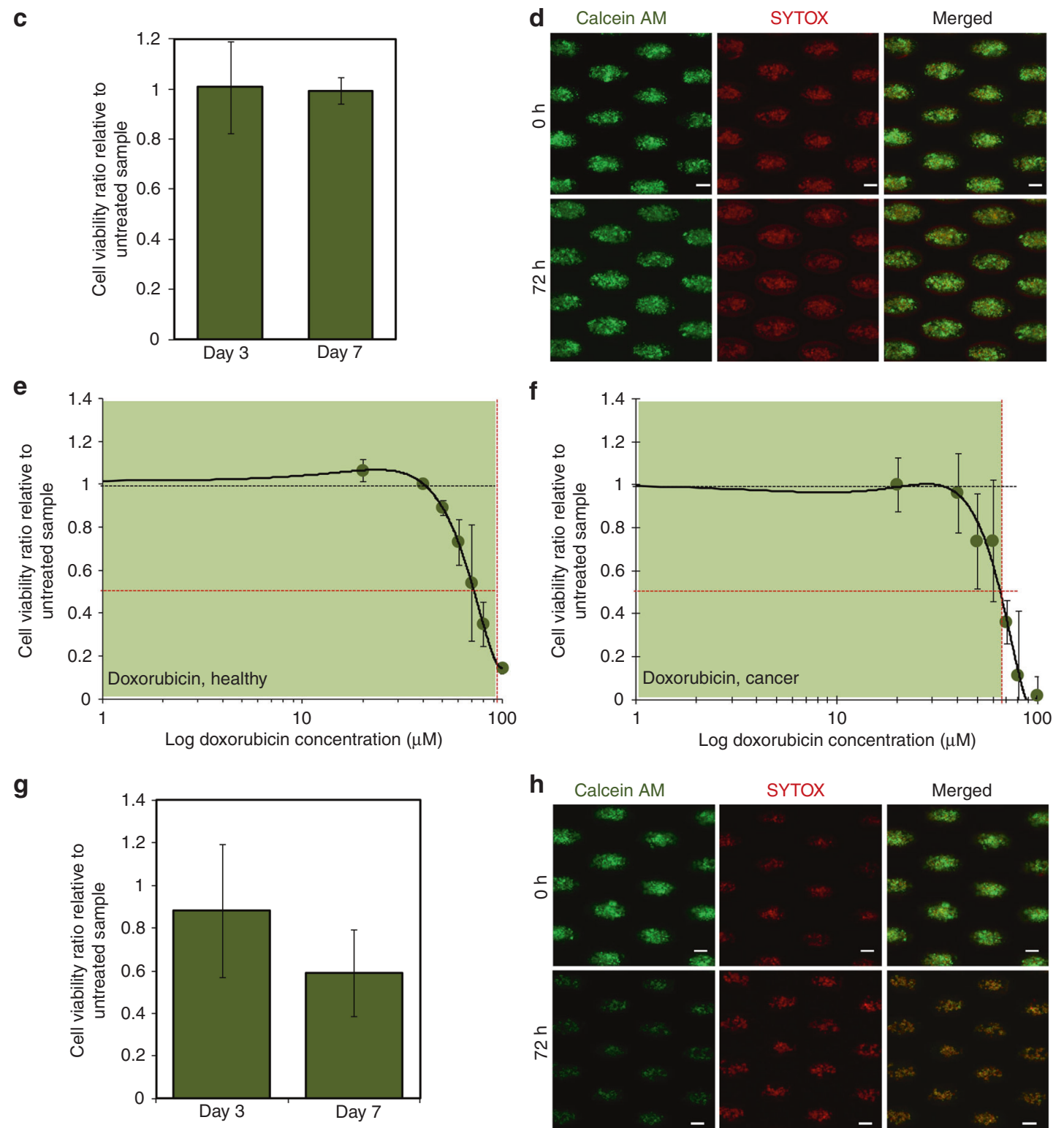

Viability ratios of cultures with respect to samples treated only with $0.5 \mathrm{D}$ were obtained to determine the effects of combinatorial treatment with aspirin. Vehicle samples were subjected to the same DMSO concentration as those under respective drug treatments. Under combinatorial DA treatment, viability

h
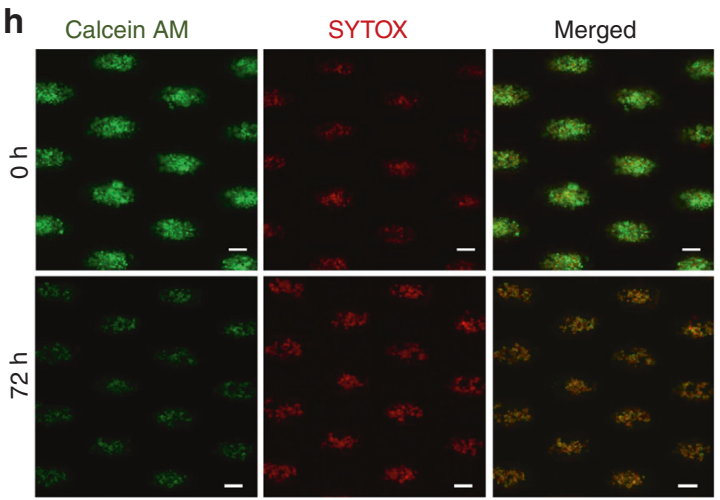

proportions remained relatively constant after short-term exposure $(72 \mathrm{~h})$ to different concentrations of aspirin in combination with $0.5 \mathrm{D}$ for both healthy and cancer cell cultures (Fig. 3a, b). However, after 7 days of exposure with combinatorial DA treatment, viability ratio, normalised to that of untreated, was 
Fig. 2 Assay outcome for single drug exposure using aspirin or doxorubicin. Viability ratios of a healthy and b MDA-MB-231 cancer cell cultures after $72 \mathrm{~h}$ drug exposure under the different percentage of aspirin concentrations $(0-200 \mathrm{mg} / \mathrm{ml})$, relative to averaged viability percentage of untreated cultures (i.e., $0 \mathrm{mg} / \mathrm{ml}$ aspirin concentration). Blood cells and cell lines serve as controls for components of the clinical patient samples, mainly white blood cells and cancer cells, respectively. Black dotted reference line indicates the averaged viability of the vehicle groups.(c Viability rates remained relatively constant after $72 \mathrm{~h}$ or 7 days of exposures to $250 \mathrm{mg} / \mathrm{ml}$ aspirin. $\mathbf{d}$ Representative images of live/dead staining of untreated cluster cultures (top) and cultures after $72 \mathrm{~h}$ treatment (bottom). Scale bar is $100 \mu \mathrm{m}$. The viability ratio of e healthy and f MDA-MB-231 cancer cell cultures after $72 \mathrm{~h}$ drug exposure with different percentage of doxorubicin concentrations alone, relative to averaged viability percentage of untreated cultures (i.e., $0 \mu \mathrm{M}$ doxorubicin concentrations). The inhibitory concentration at $50 \%$ viability (IC50) values (horizontal dotted line) was obtained based on the corresponding drug concentration value that reflected $50 \%$ cell viability as indicated on the graphs by the vertical dotted lines (healthy cultures: $0.84 \mu \mathrm{M}$; cancer cell culture: $0.75 \mu \mathrm{M}$ ). Green regions show the concentrations selected for evaluation of long-term cultures for subsequent drug experiments. Black dotted reference line indicates the averaged viability of the vehicle groups. $\mathbf{g}$ Viability decreases after prolonged exposure to $0.5 \mu \mathrm{M}$ doxorubicin. $p=0.189$, with respect to differences in cell viability for cultures treated with doxorubicin only for 7 days and $72 \mathbf{h}$. $\mathbf{h}$ Representative images of live/dead staining of untreated cluster cultures (top) and cultures after $72 \mathrm{~h}$ treatment (bottom). Scale bar is $100 \mu \mathrm{m}$

significantly reduced to an average value of $0.35 \pm 0.05(p<$ 0.00001 ; Fig. 3c). The highest killing efficacy on Day 7 was observed at $482.5 \mathrm{mg} / \mathrm{ml}$ aspirin concentration in combination with $0.5 \mathrm{D}(p=0.039)$. Intriguingly, the ratio of apoptotic cells, normalised to that of untreated, after combinatorial DA treatment increased significantly even within $72 \mathrm{~h}$ of exposure $(63 \pm 0.1 \%, p$ $<0.00001)$ and was maximised after 7 days of exposure (83 \pm $0.06 \%, p=0.0009$; Fig. $3 \mathrm{~d}$ ), as compared with treatment with doxorubicin alone. The highest proportion of apoptotic cells was also obtained under treatment within $445-500 \mathrm{mg} / \mathrm{ml}$ aspirin concentration in combination with $0.5 \mathrm{D}$. Hence, we demonstrated that the killing efficacy of combinatorial DA therapy, especially with $0.5 \mathrm{D}$ and $445-500 \mathrm{mg} / \mathrm{ml}$ aspirin could be more effective than the killing efficacy administered by treatment with $0.5 \mu \mathrm{M}$ doxorubicin alone $(0.5 \mathrm{D})$ after 7 days of exposure (Fig. 3e-h) (Figure S7A). Killing efficacy of DA treatment significantly surpassed that of treatment with doxorubicin alone after 7 days of exposure, as determined by the live and apoptotic cell proportions ( $p<0.01$; Fig. 3g, h) (Figure S7B).

Combinatorial anti-inflammatory therapy reduced gene and protein expressions associated with the formation of cancer stem cells

It has been reported that secondary tumours in cases of relapse after treatment are often multidrug-resistant. ${ }^{40}$ This observation may be attributed to an incomplete eradication of heterogeneous cell populations under targeted anti-cancer therapeutic strategies. ${ }^{41}$ Here, we support this observation by demonstrating an increase in proportion in $\mathrm{CD} 44^{+} / \mathrm{CD} 24^{-}$phenotype cells after exposure of cancer cell clusters to doxorubicin. Prior studies have also demonstrated that an increase of $\mathrm{CD} 44^{+} / \mathrm{CD} 24^{-}$phenotypes in epithelial-like luminal breast cancer subtypes lead to a heightened resistance to chemotherapy. ${ }^{42}$ The $\mathrm{CD} 44^{+} / \mathrm{CD} 24^{-}$ phenotype corresponded to the proportion of CSC-like cells, ${ }^{43}$ and they were identified by immunostaining of the harvested cluster cultures after 7 days of exposure to $0.5 \mu \mathrm{M}$ of doxorubicin (Fig. $4 \mathrm{a}$, b) (Figure S8). Direct cohort measurements were obtained instead of conventional methods such as fluorescence-assisted cell Sorting, to prevent loss of target cells in these heterogeneous populations. Increase in CSCs post treatment is undesirable as they have a high potential to form secondary metastases, leading to cancer relapse. ${ }^{44}$

Setting the averaged ratio of $\mathrm{CD} 44^{+} / \mathrm{CD} 24^{-} \mathrm{CSCs}$ generated upon treatment with $0.5 \mu \mathrm{M}$ of doxorubicin alone as 1 and untreated samples as $0.058 \pm 0.008$, we assessed the proportion of CSCs in combinatorial DA-treated samples (Fig. 4c). CSC pool size varied with treatment condition, ranging from 5.5 to $26.3 \%$ in treated cohorts. Untreated cohorts had a CSC pool size of below $1.2 \pm 0.02 \%$. Combinatorial DA treatment with $250 \mathrm{mg} / \mathrm{ml}$ aspirin reduced the proportion of CSCs slightly (ratio: $0.93 \pm 0.33$, $72 \mathrm{~h}$ ) but at combinatorial DA treatment with $500 \mathrm{mg} / \mathrm{ml}$ aspirin resulted in a significant reduction of CSC proportion to a ratio of
$0.57 \pm 0.13(72 \mathrm{~h})$. The reduction in CSC levels of cultures was much significant than the overall reduction in cellular viability (Fig. $3 \mathrm{c}$ ) over $72 \mathrm{~h}$ or 7 days treatment, hence the reduction of CSC cohorts under combinatorial DA treatment was not a result of the selective killing of non-CSCs (Figure S8). The overall CD44 expression was also significantly lower under combinatorial DA treatment as compared with single doxorubicin treatment (Figure S9). Hence, under combinatorial DA therapy, the averaged proportion of CSC-like phenotype was reduced over time with increasing doses of aspirin (Fig. 4d). Despite the overall change in $\mathrm{CD} 44^{+} / \mathrm{CD} 24^{-}$phenotype proportions, expression levels of CD44 in $\mathrm{CD}_{4}{ }^{+}$cells did not seem to vary significantly (Figure S10).

When treated cells were transferred to a new microwell-based cluster assay to determine cluster-forming potential, ${ }^{34}$ combinatorial DA treatment $(250 \mathrm{mg} / \mathrm{ml} \mathrm{A}+0.5 \mathrm{D})$ led to a significant reduction (0.22:1 ratio) in cancer cell cluster-forming capability as compared with treatment with doxorubicin alone ( $p<0.01$, Fig. 4 e). The known reported percentage of colony-forming cells in untreated MDA-MB-231 is < $5 \%$ (0.05 ratio). ${ }^{45,46}$ Intriguingly, treatment with aspirin alone was not expected to influence cluster formation, yet a higher cancer cell cluster formation potential (3.57:1 ratio) was observed for single aspirin treatment $(0.5 \mathrm{~A})$ as compared with combinatorial DA treatment $(p=0.037)$. A similar trend was observed when treated cells were transferred to three-dimensional agarose gels for spheroid formation, and the proportion of cells forming spheroids was significantly lowest in combinatorial DA-treated cells and highest in cells treated with doxorubicin only ( $p<0.01$, Fig. $4 \mathrm{f}$, Figure S11).

Heterogeneous response of therapy in clinical cohorts highlights the importance of patient-derived preclinical models

We processed 68 clinical blood samples from patients with breast cancer using the preclinical CTC Cluster Assay to obtain positive cultures for combinatorial drug treatment evaluation. The assay demonstrated high efficiency of cluster formation, of which $60.9 \%$ of the samples demonstrated cluster positivity. This varied with treatment time point, with $72.0 \%$ positivity in baseline samples but $54.9 \%$ positivity in post treatment samples (Table S1). Of the $60.9 \%$ of positive samples, 17 samples were evaluated due to a minimum cell count per sample required for seeding of multiple channels to allow parallel evaluation of different treatment conditions (Table S2). Killing efficacy was more variable in patient-derived CTC clusters, reflecting the heterogeneity of clinical samples. In all, $62.5 \%$ of clinical samples demonstrated a higher killing efficacy with combinatorial DA treatment as compared with doxorubicin alone after $72 \mathrm{~h}$ exposure. CTCs in some clinical models were also eradicated more efficiently with doxorubicin alone (ASL36, CES93 and CES76), reflecting the heterogeneity of patient profiles and the importance for reliable patient-derived models to screen patients suitable for similar antiinflammatory and anti-cancer strategies (Fig. 5a, b). 

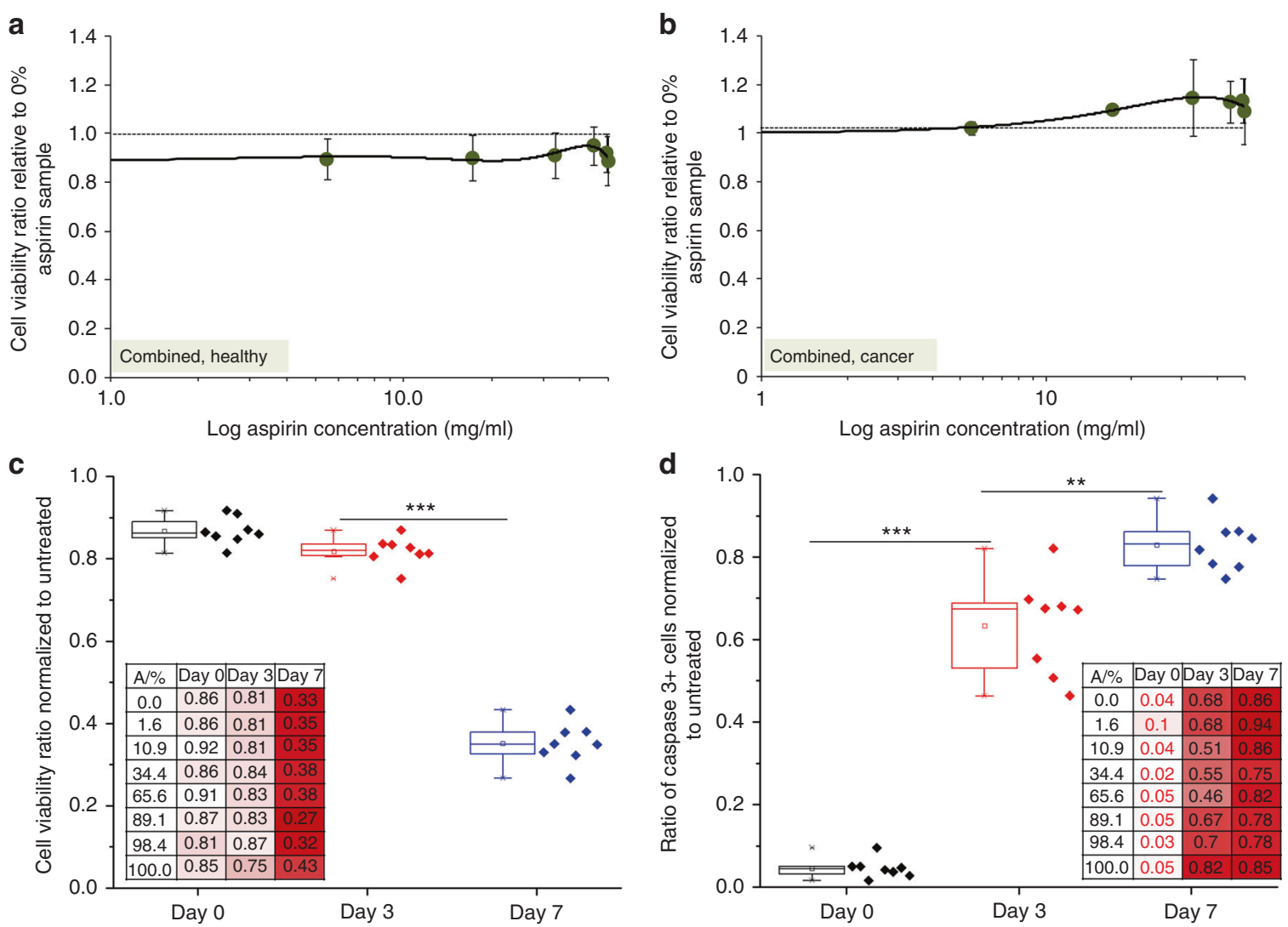

e

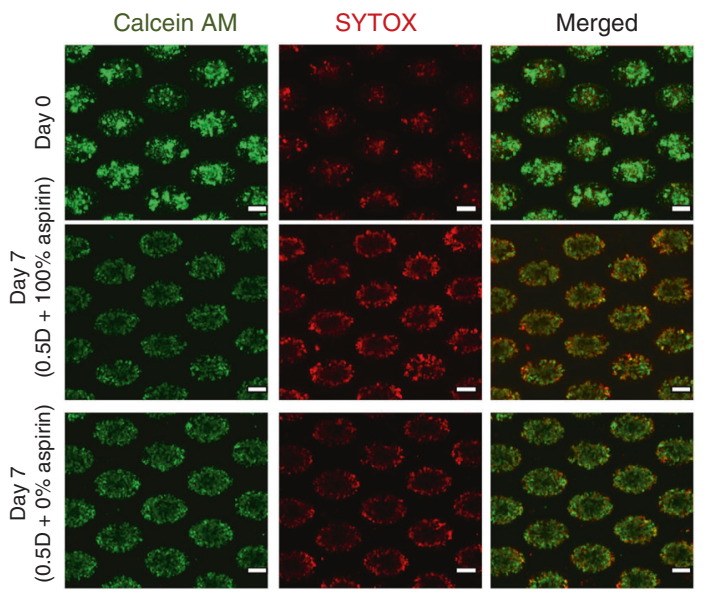

g

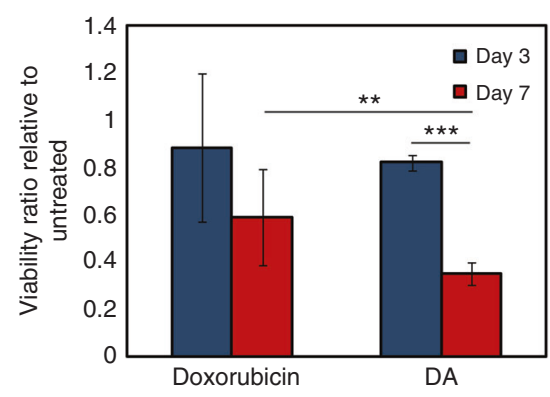

The reduction of CSCs in patient-derived CTC clusters was similarly varied across the cohort owing to the heterogeneity of clinical samples. $64.7 \%$ of clinical samples demonstrated a higher reduction in CSC proportions upon exposure to combinatorial DA

\section{f}

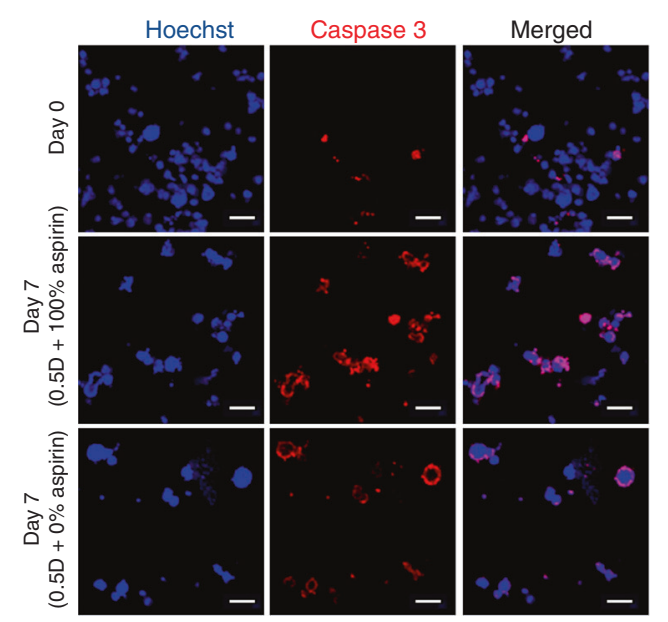

h

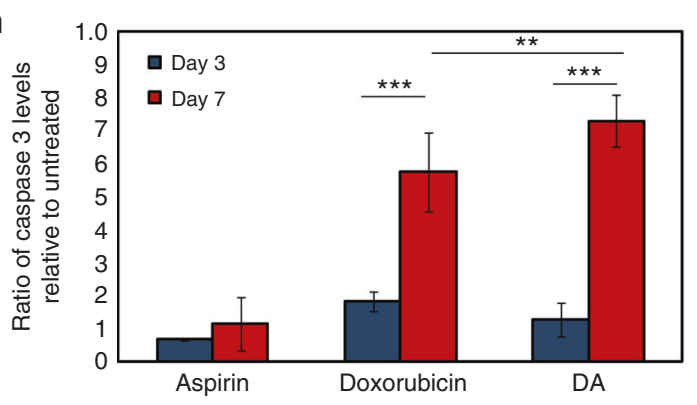

treatment, as compared with doxorubicin alone. Some clinical samples demonstrated a reduction in CSCs even with doxorubicin alone but were further reduced under combinatorial DA treatment $(38.9 \%, 7 / 18)$ (Fig. 5 C). Some samples also reflected a significantly 
Fig. 3 Viability rates under Combinatorial DA treatment administration. The viability ratio of a healthy and b MDA-MB-231 cultures after $72 \mathrm{~h}$ drug exposure to combinatorial DA treatment with different concentrations of aspirin only and fixed concentrations of doxorubicin at $0.5 \mu \mathrm{M}$ $(0.5 \mathrm{D})$, relative to the averaged viability percentage of cultures treated only with $0.5 \mathrm{D}$. Black dotted reference line indicates the averaged viability of the vehicle groups. c Viability ratio decreases after prolonged exposure to combinatorial DA treatment. Viability proportion varied with aspirin concentration under combinatorial therapy (inset). ${ }^{* *} p<0.00001$. d The proportion of apoptotic cells (caspase-3 positive) increases after prolonged exposure to combinatorial DA treatment. Viability proportion varied with aspirin concentration under combinatorial therapy (inset). ${ }^{* * *} p<0.00001,{ }^{* *} p<0.01$. e Representative images of cancer cell clusters stained with live/dead dyes of untreated cluster cultures (top) and cultures after $72 \mathrm{~h}$ treatment (bottom). Scale bar is $100 \mu \mathrm{m}$. f Representative images of resuspended single cells from cluster cultures, stained with Caspase-3 antibody and nuclei dye. Scale bar is $20 \mu \mathrm{m}$. Killing efficacies of drug treatment with doxorubicin alone and combinatorial DA treatment via $\mathbf{g}$ comparison of viable cell proportions and $\mathbf{h}$ comparison of Caspase- $3^{+}$cell proportions. ${ }^{* * *} p<0.00001$,

better response under treatment with doxorubicin only but not with combinatorial DA treatment (CES76 and BC239). One clinical sample (BC249), which was susceptible to both forms of treatment $(n=3)$ had corresponding tumour analysis demonstrating oestrogen positivity (ER+) (Table S2), and patients with ER+ subtype were widely reported to have a better overall survival. ${ }^{47}$ Overall, $41.2 \%$ of clinical samples reflected heightened treatment efficacy under combinatorial treatment only in terms of both killing efficacy and CSC reduction, reflecting the cohort that was most suitable for anti-inflammatory treatments.

Combinatorial DA treatment revert cells to a less metastatic phenotype via suppression of signalling pathways involved in the EMT

An inhibition of COX enzymes is often attributed to the mechanism of aspirin. ${ }^{48}$ In non-neoplastic studies, low doses of aspirin could inhibit COX-1 for antithrombotic effects, and higher doses (> $650 \mathrm{mg}$ per day) led to inhibition of both COX-1 and COX2 for analgesic and antipyretic effects. ${ }^{49}$

COX enzymes are often unregulated in many cancers and are associated with inflammation. ${ }^{50}$ However, the exact mechanisms of these proposed anti-cancer properties of aspirin are much less understood. Prior reports have suggested that the inhibition of COX-2 may be key for its anti-cancer therapeutic effects. ${ }^{51}$ Yet, the observations remain inconclusive as alternative mechanisms independent of COX enzymes have also been suggested for the anti-cancer effects of aspirin. ${ }^{52,53}$

We found that COX-1 protein expression was higher in both doxorubicin-treated and DA-treated cells, relative to untreated or aspirin-treated cells as determined by immunostaining $(p<0.01$, Fig. $6 a, b)$. There was also a significantly higher expression of the COX-1 protein in doxorubicin-treated cells as compared with untreated cells $(p<0.01$, Fig. $6 \mathrm{~b})$. Similar to protein expression, COX-1 enzyme activity levels were also higher in combinatorial DA-treated cells as compared with cells treated with doxorubicin only (Fig. 6c), as demonstrated by the COX-1 activity assay. Interestingly, COX-2 protein expression was reduced under combinatorial DA treatment, as evident by immunostaining (Fig. $6 \mathrm{~d}$ ). This is important as the reduction of COX-2 is directly associated with malignancy and resistance. ${ }^{54}$ Reduction in COX-2 protein expression was significantly greater under low-dose aspirin $(200 \mathrm{mg} / \mathrm{ml})$, as compared with higher dosages $(300 \mathrm{mg} /$ $\mathrm{ml}$ aspirin $(p<0.01)$ and $500 \mathrm{mg} / \mathrm{ml}$ aspirin $(p<0.00001))$ of aspirin in combinatorial treatments (Fig. 6e). These supported the notion that combinatorial DA treatment is more effective in its anticancer effects.

Cancer cells usually present as a hybrid phenotype and express intermediate levels of epithelial (E) and mesenchymal (M) characteristics. In this consideration, cancer cells are highly dynamic and can harvest benefits of both $E$ (e.g., increased adhesion) and $M$ phenotypes (e.g., stemness, resistance) to different degrees. Here, we found that there were also changes in their proportions of epithelial phenotypes after treatment with both doxorubicin alone and combinatorial treatments, as evident by the immunostaining for markers such as cytokeratin (CK) and Vimentin (Vim) on harvested cells or cell clusters in situ (Fig. 6f). CK is a cytoplasmic marker for epithelial phenotypes while Vim is a cytoplasmic marker for mesenchymal phenotypes. ${ }^{55}$ Specifically, the proportion of epithelial-like $\left(\mathrm{CK}^{+} \mathrm{Vim}^{-}\right)$cells was reduced after doxorubicin, which was most evident after 7 days of treatment. This observation was reversed under combinatorial DA treatment, with the increase of epithelial-like $\left(\mathrm{CK}^{+} \mathrm{Vim}^{-}\right)$cell populations. Under combinatorial DA treatment using a range of $7.8-328.1 \mathrm{mg} / \mathrm{ml}$ aspirin, the proportion of cells with epithelial-like phenotypes were increased within $72 \mathrm{~h}$. This was more apparent after 7 days in treatment, with a notable increase of epithelial-like phenotypes in cells treated with combinatorial DA treatment using $>171.5 \mathrm{mg} / \mathrm{ml}$ of aspirin, along with a relative increase in intermediate EMT $\left(\mathrm{CK}^{+} \mathrm{Vim}^{+}\right)$subtypes (Fig. 6f, g) (Figure S12). Clusters also demonstrated the highest packing density in untreated cultures and were lowest in doxorubicin only treated cultures (Figure S13). The overall shift toward a more E phenotype is important as it suggests a reduction in stemness and drug resistance capabilities, an observation that was supported as well with the cluster and spheroid-forming assays (Fig. 4e, f).

The IL- 6 inflammatory loop has also been associated with anticancer drug resistance through the expansion of CSCs. ${ }^{56,57} \mathrm{We}$ demonstrated that the relative IL- 6 expression as determined by ELISA normalised to the proportion of viable cells was reduced after $72 \mathrm{~h}$ treatment with doxorubicin only, but not combinatorial DA treatment ( $p<0.00001$, Fig. 6h). Interestingly, untreated cancer cells maintained as clusters over 7 days also demonstrated a reduction in IL-6 expression ( 9.6 fold at Day 7 as compared with $72 \mathrm{~h}$ ). The increase in IL- 6 was matched by the corresponding increase in JAK1 gene expression, albeit we did not detect STAT3 expression in cultures under all conditions (Figure S14). Despite the higher IL- 6 and JAK1 gene levels, the cytokines were not able to induce similar expansion of CSCs owing to inhibition of IL-6 under combinatorial treatment. Such inhibition of IL-6 under lowdose aspirin has been previously reported in both healthy and cancerous tissues. ${ }^{29,30}$ The inhibition of IL- 6 receptors while maintaining IL-6 levels is important as IL-6 is reported to demonstrate anti-inflammatory properties and play important roles in metabolism. ${ }^{58,59}$ Disruption of the IL-6 gene results in increased systemic inflammation and reduced glucose tolerance. $^{60,61}$ Hence, a delay of IL- 6 inhibition under proposed combinatorial DA treatment could actually be beneficial and reduce 'stressful' stimuli post treatment that drives cancer cells to become more metastatic and drug-resistant. ${ }^{5,62,63}$

As Calcein AM is also an indicator of intracellular oxidative activity, ${ }^{64}$ we compared the relative Calcein AM staining intensity between untreated samples and samples treated only with aspirin, as well as combinatorial DA-treated samples with samples treated only with doxorubicin. Calcein AM intensity was higher in untreated samples (both cluster and 2D cultures) as compared with samples treated with aspirin only. Similarly, Calcein AM intensity was higher in samples treated with doxorubicin only (both cluster and 2D cultures) as compared with samples under combinatorial DA treatment (Figure S15). The differential staining intensity of Calcein AM after 7 days of treatment suggests a reduction in intracellular oxidative activity in samples under both 
a
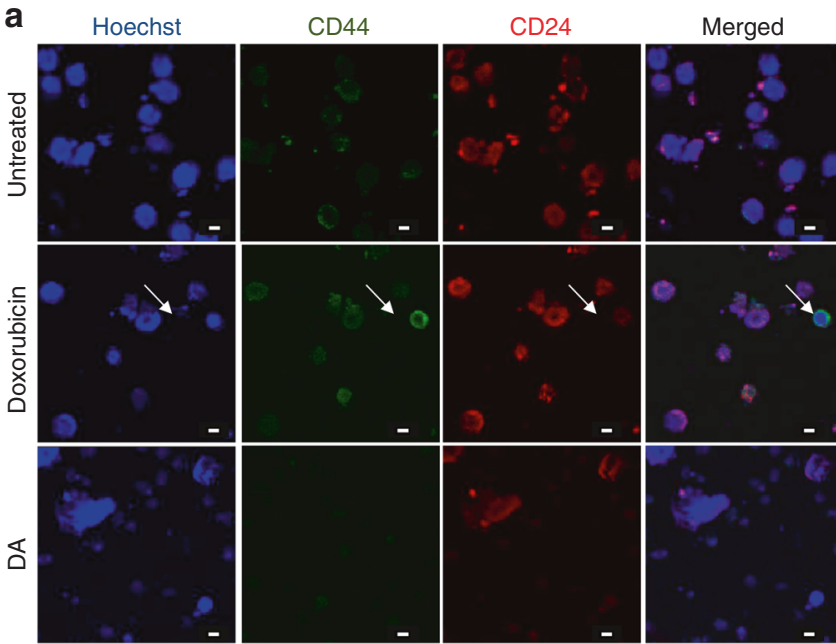

C

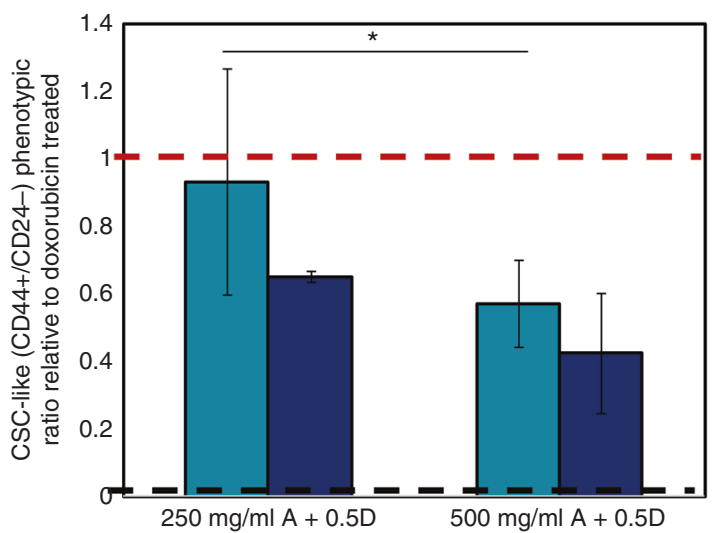

e

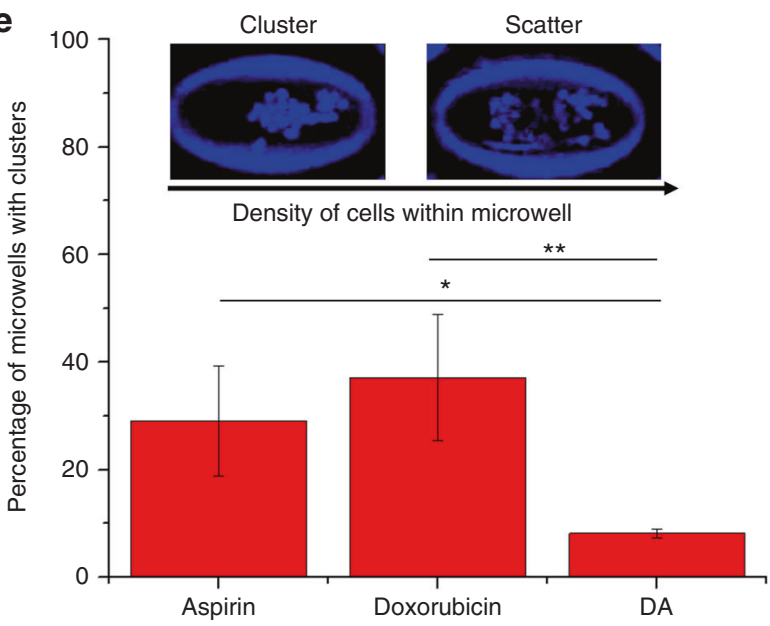

b
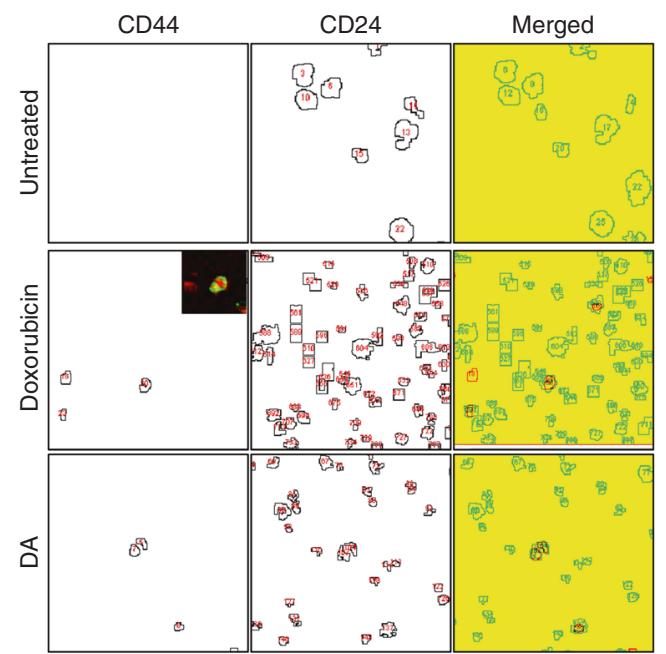

d

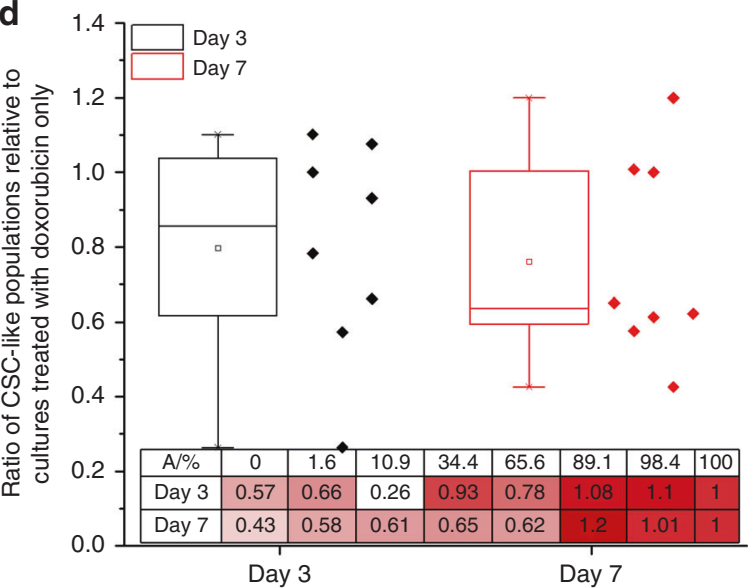

f

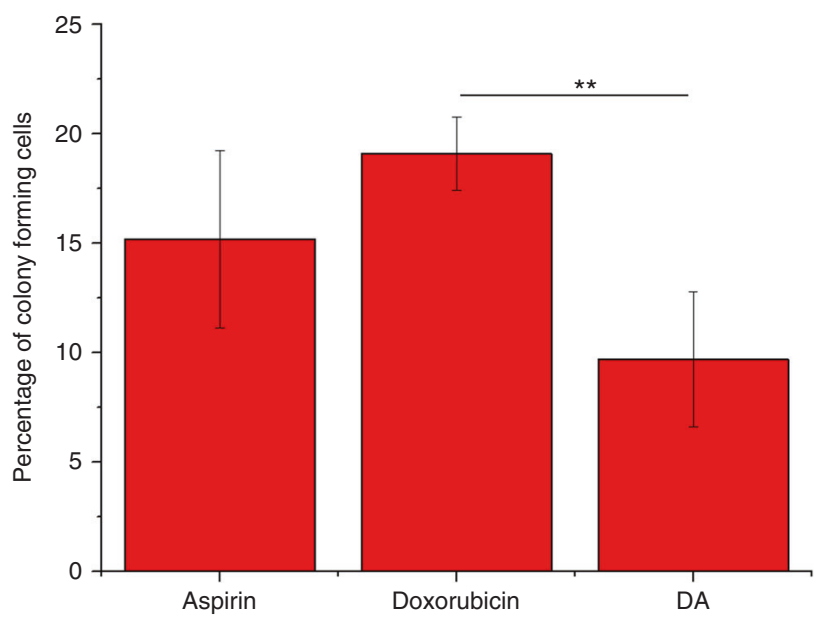

Fig. 4 Combinatorial DA treatment reduced the proportion of cancer stem cells. a Representative images of cancer cell clusters stained with CD44 and CD24. Scale bar is $20 \mu \mathrm{m}$. Doxorubicin concentration was $0.5 \mu \mathrm{M}$ and combinatorial DA treatment was $500 \mathrm{mg} / \mathrm{ml}$ aspirin with $0.5 \mu \mathrm{M}$. b Images of CD44/CD24 stained cells after processing with image analysis software for quantification of CD44 ${ }^{+} / \mathrm{CD}^{-} 4^{-}$cell $^{-}$ proportions. Doxorubicin concentration was $0.5 \mu \mathrm{M}$ and combinatorial DA treatment was $500 \mathrm{mg} / \mathrm{ml}$ aspirin with $0.5 \mu \mathrm{M}$. c Relative proportion of CD44 ${ }^{+} / \mathrm{CD} 24^{-}$cells under DA treatment with low-dose $(\sim 327.5 \mathrm{mg} / \mathrm{ml})$ or high-dose $(\sim 500 \mathrm{mg} / \mathrm{ml})$ aspirin concentrations generated in the assay. Black and red dash lines indicate average proportions of CSC in untreated and cells treated only with doxorubicin respectively. ${ }^{*} p<0.05$ $(p=0.022)$. d Box plot demonstrating the suppression of CSC-like phenotype $\left(C D 44^{+} / C D 24^{-}\right)$with respect to doxorubicin exposure alone. (e) The proportion of microwells with cancer cell clusters after treatment was the lowest under combinatorial DA treatment. Cluster phenotype was determined by the density of cells per unit area. ${ }^{72} p<0.05(p=0.037),{ }^{* *} p<0.01$. f Plot demonstrating reduced colony formation ability in agarose gels when cultures were exposed to combinatorial treatment relative to single drug administration. (Top) Representative images of a stained cancer cell cluster (Calcein AM/Hochest) in agarose gel. ${ }^{* *} p<0.01$ 


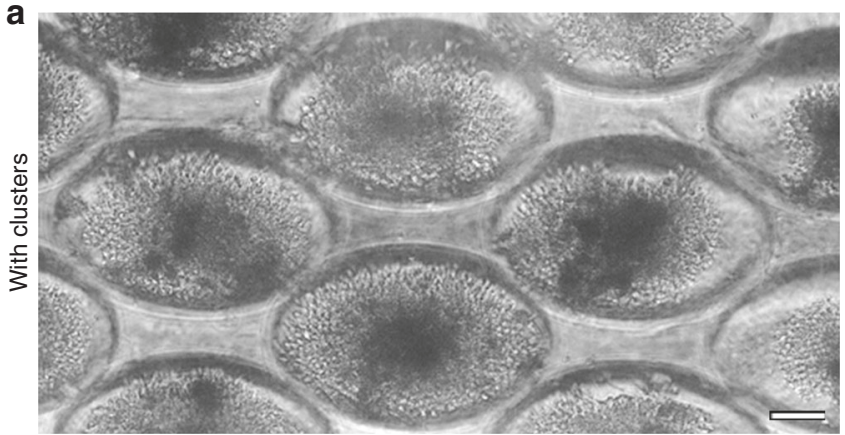

b

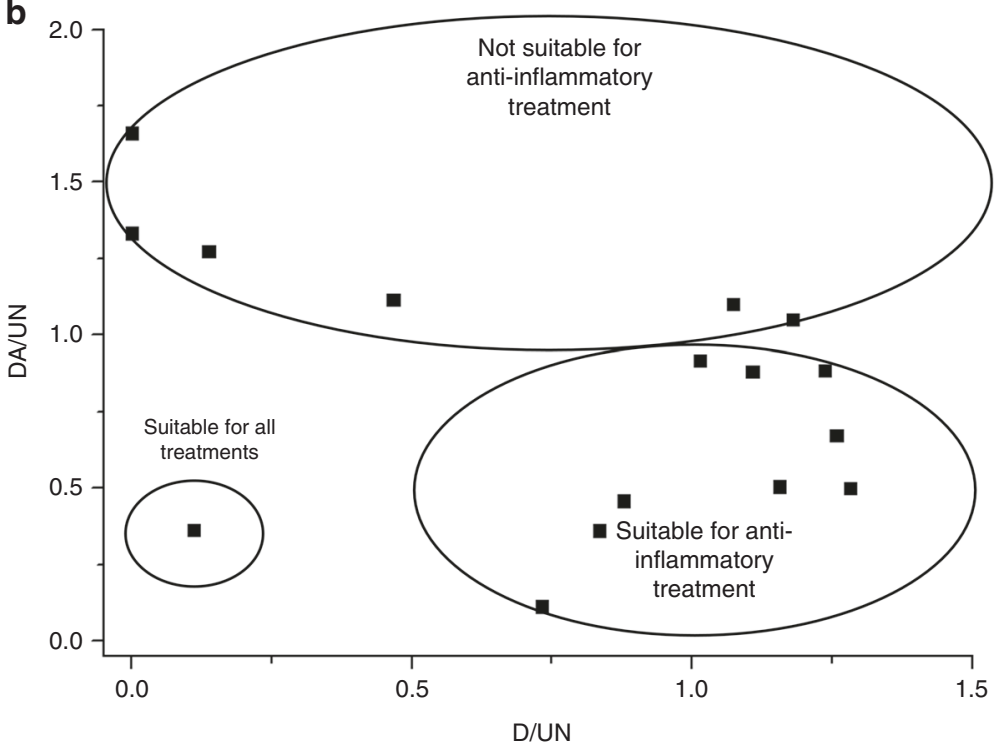

d

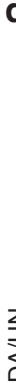

d

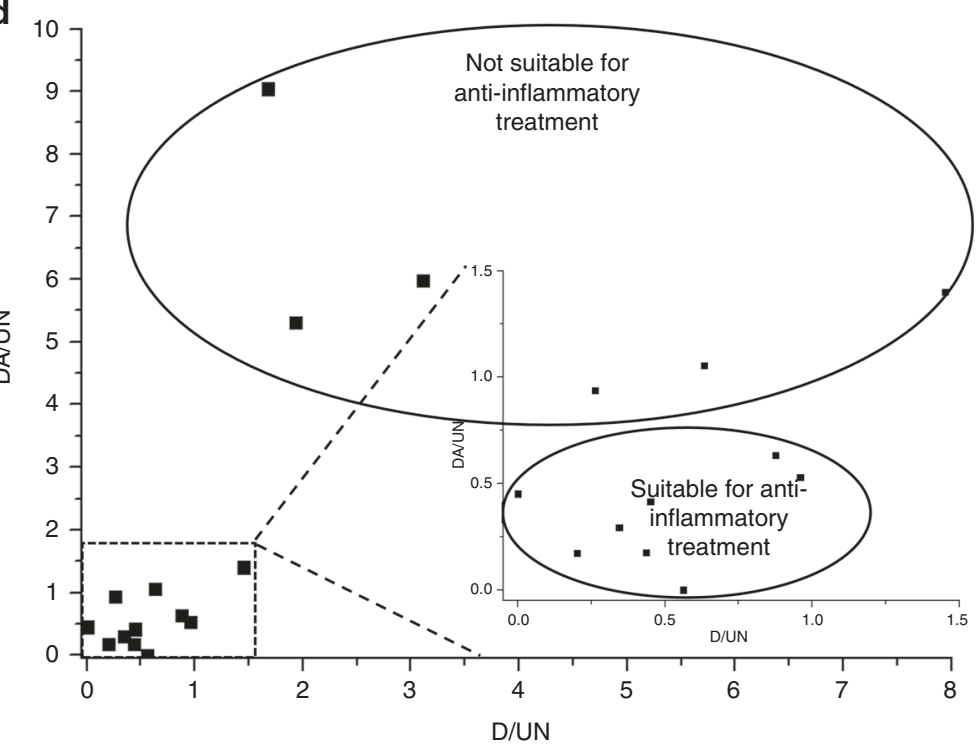

\begin{tabular}{|c|c|c|c|}
\hline \multicolumn{2}{|c|}{$\begin{array}{l}\text { Suitable for anti- } \\
\text { inflammatory } \\
\text { treatment }\end{array}$} & \multicolumn{2}{|c|}{$\begin{array}{l}\text { Not suitable for } \\
\text { anti-inflammatory } \\
\text { treatment }\end{array}$} \\
\hline SL32 & 0.39056 & CES105 & 1.02654 \\
\hline $3 \mathrm{C} 234$ & 0.15439 & CES093_2 & 1.33 \\
\hline 3C249 & 0.43289 & CES76 & 1.66 \\
\hline BC241 & 0.43708 & ASL36 & 2.39023 \\
\hline BC239 & 0.52311 & CES102 & 3.27325 \\
\hline CES64 & 0.71535 & CES093_1 & 9.2865 \\
\hline CES78 & 0.78291 & & \\
\hline CES16 & 0.79557 & & \\
\hline CES83 & 0.89144 & & \\
\hline $3 \mathrm{C}$ & 0.90546 & & \\
\hline
\end{tabular}

e

\begin{tabular}{|c|c|c|c|}
$\begin{array}{c}\text { Suitable for anti- } \\
\text { inflammatory } \\
\text { treatment }\end{array}$ & \multicolumn{2}{|c}{$\begin{array}{c}\text { Not suitable for } \\
\text { anti-inflammatory } \\
\text { treatment }\end{array}$} \\
\hline ASL32 & 0.7354 & CES76 & 1.66667 \\
\hline BC234 & 0 & BC247 & 1.92677 \\
BC241 & 0 & CES64 & 2.75389 \\
\hline CES105 & 0.40278 & CES093_1 & 3.56954 \\
\hline CES16 & 0.45 & BC240 & 5.3913 \\
\hline CES78 & 0.55126 & BC239 & 27.27 \\
\hline CES102 & 0.72186 & & \\
\hline CES093_2 & 0.8547 & & \\
\hline ASL36 & 0.85981 & & \\
\hline BC249 & 0.923 & & \\
CES83 & 0.96504 & & \\
\hline
\end{tabular}

single drug aspirin and combinatorial DA therapy. Intracellular oxidative activity is a factor in metabolism and is a key regulating process for several core functions including cell proliferation and transcription. ${ }^{65}$ We further substantiated the drop in oxidative stress levels in cells under combination therapy using the peroxidase assay and MTT assay (Figure S16). Both MTT assay and peroxidase assay reveals a reduction in absorbance levels for cultures under combinatorial treatment even within $72 \mathrm{~h}$ of treatment, which may become more significant with prolonged treatment periods.

We further speculated the possibility of combinatorial DA therapy acting through the formation of a new compound formed in the process. Although high-performance liquid chromatography (HPLC) analysis of a DA mix shows a co-elution of compounds 
416

Fig. 5 Evaluation of Combinatorial DA treatment on patient-derived CTC models using the Cluster assay. a Representative images of microwell array with clusters (left) and without clusters (right). Scale bar is $50 \mu \mathrm{m}$. b Scatter plot demonstrating the killing efficacy in clinical cohorts. DA/UN = viability of putative CTCs in patient-derived models after $72 \mathrm{~h}$ of treatment under combinatorial DA treatment, relative to untreated samples. D/UN = viability of putative CTCs in patient-derived models after $72 \mathrm{~h}$ of treatment under treatment with doxorubicin alone, relative to untreated samples. The response in terms of killing efficacy is heterogeneous with samples of comparable response grouped in black. c List of patients suitable or unsuitable for anti-inflammatory combinatorial DA treatment based on viability ratio. Patient samples that responded in terms of both killing efficacy and CSC reduction are marked in red. d Scatter plot demonstrating the reduction of CSCs in clinical cohorts. DA/UN = CSC counts of putative CTCs in patient-derived models after $72 \mathrm{~h}$ of treatment under combinatorial DA treatment, relative to untreated samples. $D / U N=$ CSC counts of putative CTCs in patient-derived models after $72 \mathrm{~h}$ of treatment under treatment with doxorubicin alone, relative to untreated samples. The response in terms of reduction of CSCs is heterogeneous with samples of comparable response grouped in black. e List of patients suitable or unsuitable for anti-inflammatory combinatorial DA treatment based on CSC counts. Patient samples that responded in terms of both killing efficacy and CSC reduction are marked in red

2.1 and 2.2, further MS, nuclear magnetic resonance (NMR) and Carbon, hydrogen, nitrogen, sulphur (CHNS) elemental analysis show that both compounds were similar to doxorubicin $(\mathrm{m} / \mathrm{z}$ spectrum $(\mathrm{M}+\mathrm{H})+$ of compounds 2.1 and 2.2: 544.17, 544.18) and were likely to be impurities. Hence the therapeutic effects appear to be the result of the combination of aspirin and doxorubicin, instead of formation of a new compound (Figure S17).

\section{DISCUSSION}

The benefits of anti-cancer combinatorial treatments have been proposed and observed for more than five decades. Yet, there is no absolute formula that can be fully effective against this disease. Such issues are largely hampered by the lack of well-defined procedures to analyse the heterogeneous responses of cancer cells to treatment and the mechanisms by which these single drugs work under combination regimens. Here, we demonstrated the use of a microfluidic assay for the analysis of combinatorial drug treatment efficacy on cell clusters cultured in 3D. This approach seeks to provide an assay for the potential screening of novel combinatorial drug regimens on cancer cell lines, and for clinical CTC clusters that can be formed with liquid biopsies from patients before or during treatment administration.

Current literature, mainly from observational studies, is not able to warrant a firm recommendation of the use of aspirin for reducing cancer risk, owing to the inconsistent results. These inconsistencies could be due to unreported behaviour factors or pre-existing conditions, statistical power, cohort selection or varying drug exposure conditions. ${ }^{25}$ It will be challenging to initiate widespread human clinical trials to iron out these issues, owing to the need for a large cohort size and procedures required to allow prolonged monitoring over time. A more viable alternative will be to put future efforts into generating in vitro models and animal models under controlled conditions to evaluate the potential toxicity and efficacy of aspirin and to determine if the use of aspirin can outweigh these risks in a patient-specific manner. Furthermore, different efficacy dosages of aspirin have been reported for various cell types, ranging from $29.3 \mu \mathrm{M}$ for chondrocytes ${ }^{66}$ to $7.3 \mathrm{mM}$ for B-chronic lymphocytic leukaemia cells. ${ }^{67}$ As a single drug supplement after anti-cancer treatment, aspirin exerts its benefits only after a latent period of 3 years (Table S2). Hence, patients are administered a high dosage of aspirin continuously, presenting a risk of drug toxicity, bleeding complications or mortality. ${ }^{68}$ Current studies suggested that the anti-cancer impact of aspirin can be highly varied with dosage and duration of intake. This contrasts with other applications of aspirin, for example, the addition of aspirin to clopidogrel beneficial for coronary syndromes is independent of aspirin dosage. ${ }^{69}$ There are also conflicting reports, which demonstrate that different aspirin dosages can reduce prostaglandin $\mathrm{E}$ levels to the same degree, ${ }^{70}$ hence conferring similar chemopreventive effects. It remains a challenge to validate the actual efficacy of aspirin with current literature, which fails to reflect underlying pre-existing conditions, statistical power, cohort selection or variations in drug exposure parameters. ${ }^{25}$ Some studies involving breast cancer even reported an inverse association of aspirin with cancer chemoprevention. ${ }^{71}$

Here, we demonstrate a heterogeneous response in terms of killing efficacy and CSC reduction, using clinical CTC cultures from liquid biopsies. CTC cultures were obtained efficiently by the cluster assay $(60.9 \%)$, with cluster-forming potential varying with treatment time points to reflect patient prognosis. ${ }^{72}$ We confirmed the enhanced cancer-killing effect (66.7\%) and reduction of CSCs $(61.1 \%)$ under combinatorial treatment for clinical CTC cultures, albeit outcomes were heterogeneous. Among the pre-treatment samples processed $(n=6)$, better response under combinatorial DA treatment was observed in samples from patients with the ER + PR-HER - tumour subtypes. Heterogeneity in clinical outcomes was widely reported in other drug studies, even in recent PD-1 targeting immunotherapy trials. ${ }^{73}$ Nonetheless, these results present an intriguing correlation between heightened drug efficacy under combinatorial DA treatment for subpopulations of breast cancer, and a larger set of clinical samples is currently being collated to validate these findings.

The main mode of action of aspirin is mediated through the inhibition of COX enzymes and subsequently their key effectors, such as PGE2. The reduction of prostaglandins, which are active lipid compounds involved in proliferation, immunity and associated with cancer metastasis, ${ }^{74}$ has been demonstrated in colorectal cancer. The beneficial effects on patients with coronary syndromes appear to act through these mechanisms. ${ }^{69}$ Specifically, COX-1 is one of the two isoforms that is irreversibly inactivated by aspirin and is part of the core mechanism involved for anti-clotting effects of platelets ${ }^{49}$ even at low dosages $(<$ $650 \mathrm{mg} /$ day). However, its anti-cancer mechanisms remain under debate, in part due to tumour heterogeneity, along with the complexity and multiplicity of aspirin's biochemical mechanisms. The inhibition of COX enzymes also affects several pathways, resulting in wide-ranging effects such as the inhibition of platelet aggregation and prevention of cardiovascular disease occurrence. ${ }^{75}$ Thus far, the anti-cancer effect of aspirin has always been explored as a single drug supplement and administered post-anticancer treatment. Furthermore, the intake of aspirin does not seem to confer consistent therapeutic benefits across different cancer types (Table S3). ${ }^{19}$ Several hypotheses have been suggested, such as the involvement of PIK3CA, which mediates PTGS2 expression, the gene that codes for COX-2. ${ }^{76}$ COX-2 inhibitors are also known to independently demonstrate potent anti-tumourigenic effects. ${ }^{77,78}$

We suggest that the anti-inflammatory effect of aspirin is exerted via inhibition of COX enzymes. ${ }^{48}$ DNA-damaging agents such as doxorubicin are known to induce expression of COX-2, in association with p53. ${ }^{79}$ The anti-cancer effects of our combinatorial treatment regime appear to be mediated by both COXdependent and COX-independent pathways, as evident by reduction of COX-2 expression after combinatorial DA treatment. COX-independent pathways are also involved as the low doses of aspirin $(<500 \mathrm{mg} /$ day) used here has little or no activity against 
a
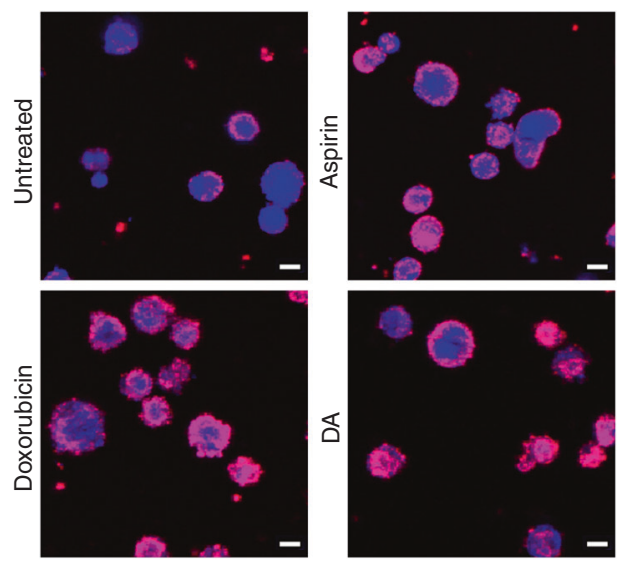

C

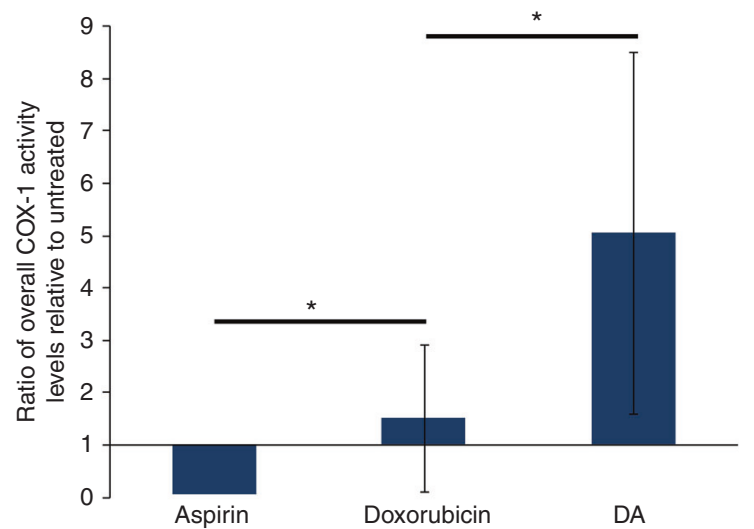

b

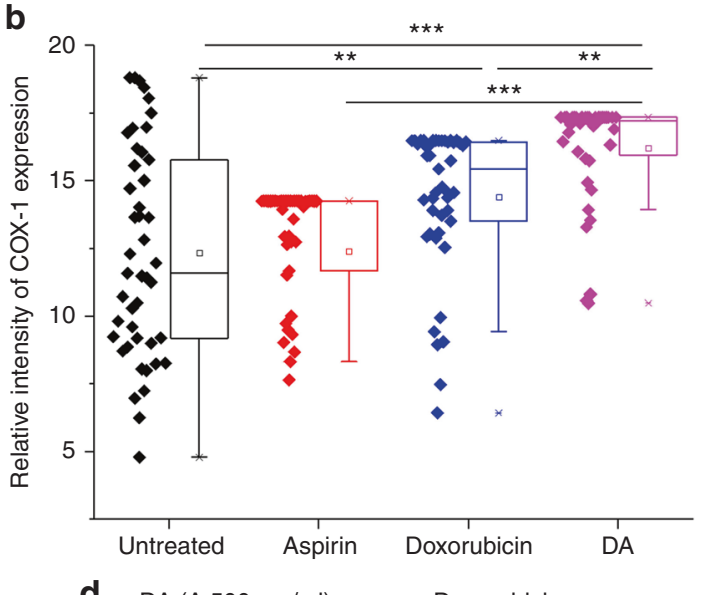

d $\mathrm{DA}(\mathrm{A}: 500 \mathrm{mg} / \mathrm{ml})$

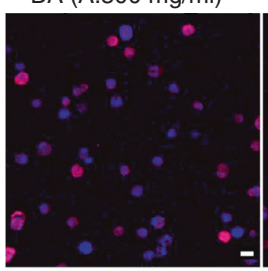

DA $(A: 300 \mathrm{mg} / \mathrm{ml})$

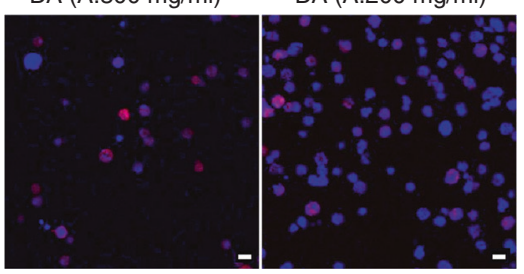

e

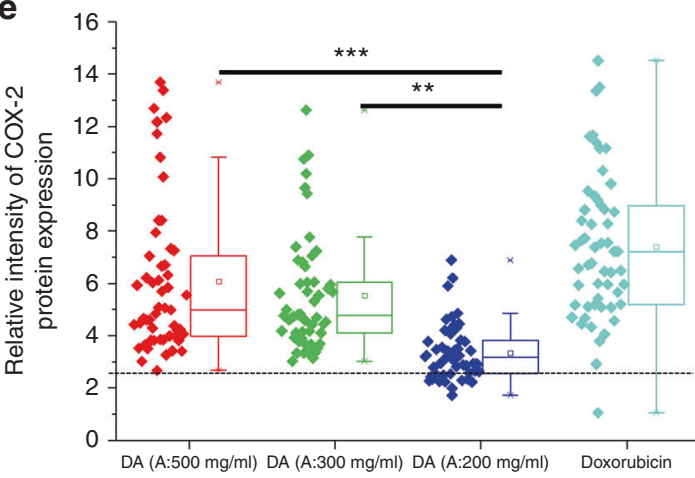

g
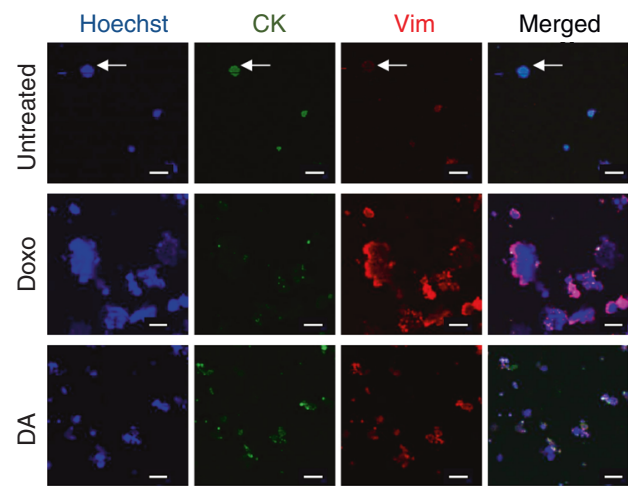

COX- $2 .^{80}$ We propose that the reduced tumour-forming potential was also mediated by the suppression of inflammatory-associated signalling pathways involved in EMT, as evident by the concurrent reduction of mesenchymal marker expression.
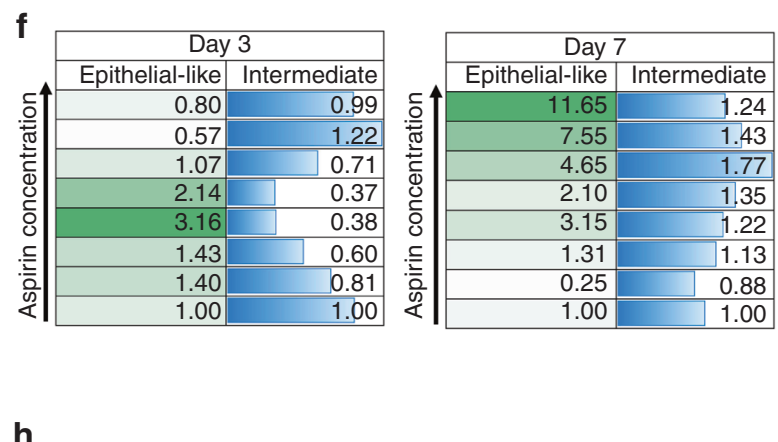

h

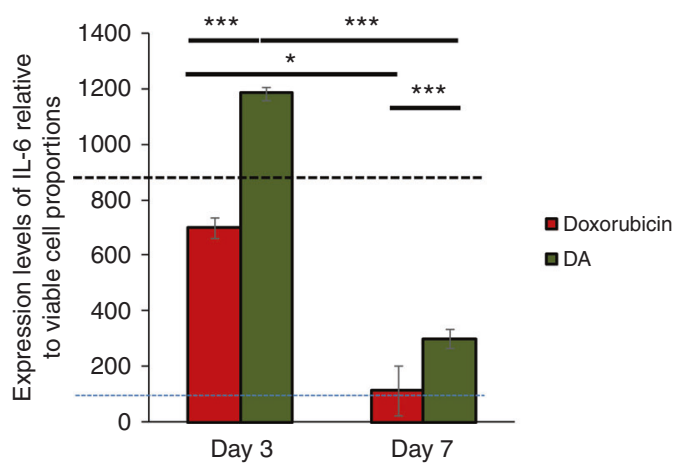

We observed a reduction in COX-2 expression under combinatorial DA treatment, albeit more apparently at low aspirin dosages (Fig. 6d, e). We are motivated to work with lower doses of aspirin $(\leq 500 \mathrm{mg} / \mathrm{ml})$ under short-term treatment and controlled 
418

Fig. 6 DA Combinatorial DA treatment acts via a COX-independent pathway and was mediated by anti-inflammatory mechanisms suppressing metastatic phenotypes. a Immunostaining for COX-1 protein on harvested cell cluster cultures. Scale bar is $10 \mu \mathrm{m}$. $\mathbf{b}$ Expression levels of COX-1 protein in cultured cells after $72 \mathrm{~h}$ of drug exposure normalised to background levels. ${ }^{* *} p<0.00001,{ }^{* *} p<0.01$. c Relative levels of COX-1 activity levels of cultured cells after $72 \mathrm{~h}$ of drug exposure, as determined by the COX-1 activity assay. Aspirin and doxorubicin: ${ }^{*} p<0.05(p=0.043)$. Aspirin and combinatorial DA: ${ }^{*} p<0.05(p=0.012)$. d Immunostaining for COX-2 protein. DA refers to combinatorial treatment with $0.5 \mathrm{D}$ and $500 \mathrm{mg} / \mathrm{ml}$ aspirin as indicated. Doxorubicin treatment refers to treatment with $0.5 \mu \mathrm{M}$ dosage of doxorubicin. Scale bar is $10 \mu \mathrm{m}$. e Relative intensity levels of COX-2 protein after 7 days of drug exposure. ${ }^{* * *} p<0.00001,{ }^{* *} p<0.01$. f Proportions of epithelial-like $\left(\mathrm{CK}^{+} \mathrm{Vim}^{-}\right)$and intermediate EMT $\left(\mathrm{CK}^{+} \mathrm{Vim}^{+}\right)$phenotypes after combinatorial DA treatment, after $72 \mathrm{~h}$ or 7 days of drug exposure. Data are relative to that of cells treated only with doxorubicin. $\mathbf{g}$ Immunostaining for CK and Vimentin on harvested cell cultures. Arrows indicate an epithelial-like cell. Scale bar is $20 \mu \mathrm{m}$. h Cytokine IL-6 levels secreted under treatment with doxorubicin only and combinatorial DA-treated cells under exposure periods of $72 \mathrm{~h}$ and 7 days, as determined by ELISA assays. The expression levels are normalised to the viable cell proportions of each culture condition. Reference lines were provided for the average expression level of IL- 6 in untreated cluster cultures after $72 \mathrm{~h}$ (black dotted line) and at 7 days treatment (the blue dotted line) respectively. ${ }^{*} p<0.05,{ }^{* * *} p<0.00001$

conditions, as patients under high dosage intakes often incur adverse reactions, such as bleeding. ${ }^{68}$ In anti-cancer studies, it was shown that low doses of aspirin $(<500 \mathrm{mg} /$ day) has little or no activity against $\mathrm{COX}-2,{ }^{80}$ an observation that was also reflected in our studies (Fig. 6e). Previous studies also explored the use of COX-2 inhibitors on reversal EMT and tumour growth inhibition in bladder cancer, but only one of three drugs tested demonstrated the desired anti-cancer effects. ${ }^{81}$ Thus, low-dose aspirin in combination with doxorubicin may promote anti-cancer properties via multiple processes dependent and independent of the COX pathway.

EMT transition is regulated by a myriad of signalling pathways. One of these is known to be triggered during inflammation and cancer and mediated by IL- 6 through Janus kinase-signal transducer and activator of transcription 3 (STAT3)-induced SNAIL1 expression. ${ }^{55}$ We also evaluated gene levels of STAT1 and STAT2 expression, known as key mediators of the classic host immune defense system (Figure S14) and noted the highest increase in STAT2 gene expression under combinatorial DA treatment as compared with other conditions. This suggests that the receptors of type 1 and type 3 IFNs may be involved, as STAT2 alone works via this pathway ${ }^{82}$ and has a unique role in cytokine signalling. ${ }^{83}$

Furthermore, it is important to study EMT phenotypes as specific subtypes such as the mesenchymal cells and CSCs are associated with key aspects of tumour progression. ${ }^{39,84,85}$ While high levels of IL-6 are commonly associated with the tumour microenvironment, IL- 6 has also been reported to demonstrate anti-inflammatory properties and play important roles in metabolism. ${ }^{58}$ Disruption of IL-6 gene had been shown to result in increased systemic inflammation and reduced glucose tolerance. ${ }^{61}$ Recent findings have also suggested crucial roles of systemic immunity and cytokine-dependent homoeostatic programmes in the effectiveness of immune-based therapeutic methods ${ }^{86}$ and tumour progression. ${ }^{87}$

This maintenance of IL-6 expression levels with combinatorial DA treatment could generate less 'stressful' stimuli post treatment, which are known to have either a prodeath or prosurvival role and could drive cancer cells to become more metastatic and drugresistant. $^{5}$ Such observations suggest that the combinatorial DA therapy induces similar suppression of inflammatory signalling pathways while reducing therapeutic stress to prevent the onset of cellular adaptations, which will lead to more malignant subtypes via EMT.

Our current hypothesis argues that the effects of the combinatorial DA treatment are likely to involve both COXdependent and COX-independent pathways, the former being evident by the reduction of COX-2 expression. Studies have demonstrated that COX-2/PGE2 pathways are potent inhibitors of EMT for epithelial cells, ${ }^{31}$ and the resultant COX-2-derived PGE2 and PGD2 are mediators of anti-EMT. ${ }^{32}$ Due to the varied outcomes under treatment with different aspirin concentrations, we propose that the combinatorial therapy with low-dose aspirin may also act via COX-independent pathways ${ }^{88}$ (Fig. 1c). This is within expectations as low-dose aspirin has been reported to have no effects on COX-2 expression, ${ }^{80}$ and various factors affecting COX expression are also reported to be involved in pathways that directly affect cancer cell behaviour (e.g., hypoxia-inducible factor$1 \mathrm{a}^{89}$ ). In fact, transforming growth factor- $\beta 1$, a multifunctional cytokine involved in various pathophysiological processes, has been reported to induce downregulation of COX-2 while independently facilitating EMT in both normal and lung cancer cells. ${ }^{90,91}$ Here, we suggest that combinatorial DA treatment is also mediated by similar anti-inflammatory pathways, which lead to less 'stressful' stimuli post treatment. Such stimuli are known to have a prodeath or prosurvival role and drive cancer cells to respond or adapt to become more metastatic and drug-resistant. ${ }^{5}$ Our method of combinatorial DA treatment hence, in turn, could reduce the onset of metastatic phenotypes. Conferring anti-cancer benefits via COX-independent pathways is of certain benefits, as the excessive suppression of some anti-carcinogenic PGEs ${ }^{92}$ through the COX-dependent pathway may induce detrimental effects that are pro-tumourigenic instead, an effect likely to be dose-dependent as well.

Overall, our findings serve as a basis for optimism regarding the utilisation of low-dose aspirin in combination with anti-cancer drugs as an effective chemopreventive therapy against breast cancer and potentially other cancer types.

\section{MATERIALS AND METHODS}

Fabrication of gradient generator and liquid barrier layer The device is the assembly of three functional layers, the bottom one containing the rounded ellipsoidal wells, the middle one with the open channels for liquid containment and the top one with the gradient generator microchannels. Each layer was produced separately in polydimethylsiloxane (PDMS), using a soft lithography approach. They were then aligned and permanently bonded via surface activation of the PDMS by $\mathrm{O}_{2}$ plasma.

The detailed description of the fabrication protocol was discussed elsewhere. ${ }^{72}$ In brief, the gradient generator and open channels layers require for standard photo-lithography (SU-8 negative tone photo-resist on a silicon wafer as substrate) for the former and metal micro-machining the latter to produce their respective primary molds. Two inlets allow the flow of a single drug or double drug combinations into the device (Fig. 1b), which will be mixed by the gradient generator component to achieve various drug combinations within the compartmentalised channels. Numbers of PDMS replicas could then be fabricated easily.

Fabrication of 8-channel and 10-channel cancer cell cluster assays The fabrication of the primary mold for the rounded wells was more complex. The size $(250 \mu \mathrm{m}$ major axis and $150 \mu \mathrm{m}$ minor axis) and depth required $(150 \mu \mathrm{m})$ does not allow for the use of a reflow strategy, ${ }^{93}$ due to lack of suitable photoresists that could be coated with the required thickness. Hence, we applied a diffuser 
back-side lithography procedure, ${ }^{94}$ which works using SU-8 negative tone photo-resist. SU-8 can be coated up to $1 \mathrm{~mm}$ thick and it results in more durable structures being negative tone.

Back-diffuser lithography works by exposing to UV-light a thick photo-resist coated on the surface of an optical mask from the back and through an optical diffuser, such as an opal diffuser plate. In this way, it is possible to define rounded volumes of exposed photo-resist. After post-baking and development, ellipsoidal pillars of hardened SU-8 remain on the mask plate and could be used as a primary mold for soft lithography. The number and distribution of these lenses were defined by the openings in the optical mask, therefore the choice of the number of channels and the density of wells for each channel can be freely defined.

Instead of using the optical plate directly as a primary mold, with the purpose of increasing its lifetime we choose to use a double-replica strategy. The first replica made in PDMS out of the optical plate was with ellipsoidal wells (same polarity as in the final device), but a second replica out of this PDMS mold was with ellipsoidal pillars, same as in the original optical plate. This last PDMS replica was finally used as a working mold for the fabrication of multiple layers with wells for the final device application.

Cell line cultures

MDA-MB-231 (HTB-22TM, ATCC, USA), a human breast adenocarcinoma cell line, was used in the formation of cancer cell clusters. SW480 (ATCC CCL-227), a colorectal epithelial cell line was used to characterise cluster formation. Cell lines were cultured in highglucose Dulbecco's modified Eagle's medium (DMEM) (Invitrogen, USA) supplemented with $10 \%$ fetal bovine serum (Invitrogen, USA) and $1 \%$ penicillin-streptomycin (Invitrogen, USA). Cultures were maintained under normoxia at $37^{\circ} \mathrm{C}$ in a humidified atmosphere and $5 \%(\mathrm{v} / \mathrm{v}) \mathrm{CO}_{2}$. Cells were cultured in sterile $25 \mathrm{~cm}^{2}$ flasks (BD Bioscience, USA) and sub-cultivated two times a week, with media refreshed every $48 \mathrm{~h}$.

Establishment and evaluation of CTC clusters from liquid biopsies Sixty-nine blood samples collected from 45 patients with breast cancer (Table S3) using EDTA-coated vacutainer tubes (BectonDickinson). Blood cells and cell lines were controls for components of the clinical patient samples, mainly white blood cells and cancer cells respectively. Within $10 \mathrm{~h}$, the blood samples were lysed with $2 \times$ red blood cell lysis buffer (G-Biosciences, MO, USA, cat. no. 786-849). Intact non-RBCs were then removed by a centrifugation step at $1000 \mathrm{rcf}$ for 5 mins. The resulting cell pellet was resuspended in $5 \mathrm{ml}$ of $1 \times$ phosphate-buffered saline (PBS) (Vivantis Inc., USA, cat. no. PB0344-1L) and centrifuged at $1200 \mathrm{rpm}$ for 3 mins. The supernatant was discarded, and the cell pellet was resuspended in $1 \mathrm{ml}$ DMEM (Thermofisher Scientific, cat. no. 11965118). Cells were quantified using an automated cell counter (Bio-Rad, CA, USA, cat. no. 786-849). A total of $2.0 \times 10^{7}$ cells were seeded in each channel of the device to allow comparison of cluster-forming potential across samples. Samples were cultured for 14 days under a hypoxic condition at $37^{\circ} \mathrm{C}$, with $300 \mu \mathrm{l}$ media change every $48-72 \mathrm{~h}$. At the end of 14 days, phasecontrast imaging of samples at $100 \times$ magnification was performed. Percentage of cluster formation was determined using Image J (Table 2). Samples with $>50 \%$ wells that contained clusters were considered positive. The remaining cultured channels were treated with a combination of $50 \%$ doxorubicin (Sigma-Aldrich, MO, USA, cat. no. D1515) and 50\% aspirin (SigmaAldrich, MO, USA, cat. no. A5376) or 50\% doxorubicin, respectively, for $72 \mathrm{~h}$.

Preparation for cell seeding

Cell monolayers were dissociated at $80 \%$ confluence using $0.01 \%$ trypsin and 5.3 mM EDTA solution (Lonza, Switzerland). Cells were seeded at a concentration of $250-500$ cells per microwell for rapid establishment of cancer cell clusters.

Maintenance of cultures on-chip

After cell seeding, the assay was placed in a humidified $150 \mathrm{~mm}$ dish and incubated under conditions stated above. Media was refreshed every $48 \mathrm{~h}$ or earlier depending on seeding concentration. The media could be replaced manually or with a syringe pump. $^{72}$

Quantifying the proportion of microwells with cluster formation Cluster phenotype was detected with a macro ran on the ImageJ processing software (Figure S18). Microwells with denser cell packing reflect lower grey values, which will be detected as clusters. Grey values of two regions from each image were obtained: (1) Background (non-microwell) and (2) Cell region (within microwell), using an image processing software (ImageJ). In microwells with clusters, groups of grey scale values obtained within the cell region will be $<50 \%$ of the average grey scale value computed from the background region, demonstrating the presence of a denser region.

Procedures for drug-screening

Doxorubicin (Sigma, cat. no. D1515) and Aspirin (Sigma, cat. no A5376) were diluted in $100 \%$ DMSO and stored as aliquots under $-20^{\circ} \mathrm{C}$. Working concentrations of drugs were freshly prepared before use. Drugs could be introduced after overnight incubation or once clusters were established. For cultures beyond $72 \mathrm{~h}$, drug solutions diluted in supplemented media were replaced every $72 \mathrm{~h}$ for up to 7 days of exposure.

For evaluating IC50 values of monolayer cultures, cells were seeded at 0.5 million cells per chamber of an eight-well plate (Ibidi $\mathrm{GmbH}$, Germany) and exposed to a gradient of drugs added manually (Figure S1).

For screening of cancer cell clusters under various drug combinations, drugs could be introduced manually or via the gradient generator component of the assay. For a generation of drug concentrations via gradient generator, working concentrations of aspirin $(500 \mathrm{mg} / \mathrm{ml})$, doxorubicin $(0.5 \mu \mathrm{M})$ and DA $(0.5 \mu \mathrm{M}$ doxorubicin $+500 \mathrm{mg} / \mathrm{ml}$ aspirin) were prepared, respectively, for syringe loading. Loaded syringes were connected to the assays via designated inlets. The media in the assay channels $(250 \mu \mathrm{l}$ for the 8-channel assay and $150 \mu \mathrm{l}$ for the 10-channel assay) were gradually replaced with a continuous influx of fluid from the syringes pumped at $100 \mu \mathrm{l} / \mathrm{min}$.

\section{Immunostaining of cancer cell clusters in situ}

The viability of cells in each channel was determined with dyes that stain for live or dead cells, specifically Calcein AM (green, $2 \mu \mathrm{M}$; Life Technologies) and Sytox (far red, Thermofisher). Ethidium bromide was not used to avoid fluorescence emission overlap with doxorubicin. Clusters were incubated for 30 mins in situ under incubation. Alternatively, samples could be stained as single cells after gentle release from microwells by pipette resuspension. Imaging was carried out with an Olympus inverted confocal microscope (Emission filters ET460/50 m and ET535/50 m; Olympus, Tokyo, Japan). Images should be obtained with the appropriate filters to reduce background signals from doxorubicin. Cells counts were obtained using ImageJ via thresholding $(\mathrm{NIH}$, Bethesda, MD).

For immunostaining with other antibodies, clusters were released from the microwells and resuspended as single cells for analysis. For membrane proteins, samples were stained directly with fluorochrome-conjugated antibodies (CD44-fluorescein isothiocyanate (green; FITC) and CD24-Allophycocyanin (red; APC) (1:100, Miltenyi Biotec Asia Pacific, Singapore). For cytoplasmic proteins (Caspase-3; 1:250, cat. No. ab13847, Abcam) (pancytokeratin-FITC; 1:100, Miltenyi Biotec Asia Pacific, Singapore 
420

and (Vimentin; 1:250, DAKO), samples were fixed with 70\% ethanol for $10 \mathrm{~min}$ and stained with their respective primary antibodies, washed and stained with the appropriate secondary antibodies (anti-mouse or anti-rabbit Alexa Fluor $^{\circledR}$ 488, Abcam and Alexa Fluor $^{\circledast}$ 633, Abcam). Samples were incubated in the antibody cocktail for an hour, washed with PBS and imaged with confocal microscopy.

Profiling viability proportions

To establish the $I C_{50}$ values, $z$-stacks images of 25 microwells from each channel were obtained. Images from each stack were merged based on maximum intensity. Merged images were processed to identify maxima signals corresponding to each cell. For consistency, the microwells considered for evaluation were obtained at the same distance from the assay inlets.

\section{Profiling CSC-like proportions}

Images were obtained with confocal microscopy and processed with the ImageJ processing software. Specifically, locations of $\mathrm{CD}_{4} 4^{+}$and $\mathrm{CD} 24^{+}$cells were identified by setting a 'threshold' using ImageJ's thresholding tool. Twenty-five fields were evaluated per set ( $>15,000$ cells). Processed images were merged to locate regions with overlapping CD44 and CD24 signals (i.e., $\mathrm{CD} 44^{+} / \mathrm{CD} 24^{-}$cells). This proportion was then subtracted from the overall counts of $\mathrm{CD} 44^{+}$single positive cell populations to obtain $\mathrm{CD} 44^{+} / \mathrm{CD} 24^{-}$cell populations corresponding to the CSC-like fractions. For clinical samples, $\mathrm{CD} 45^{-} / \mathrm{CD} 44^{+} / \mathrm{CD} 24^{+}$populations were identified as the CSC cohorts.

\section{Cluster and spheroid colony assays}

Treated cultures were transferred as a single-cell suspension to a new microwell-based cluster assay to determine cluster-forming potential. ${ }^{34}$ Proportions of resultant clusters were evaluated as previously described. Similarly, for spheroid assays, single cells were mixed to a final concentration of $0.35 \%$ top agarose and added to a base layer of $0.5 \%$ solidified agarose. Spheroids were stained with Hoechst and imaged with an Olympus inverted confocal microscope (Olympus, Tokyo, Japan). All assays were maintained for 2 weeks prior to analysis.

Evaluation of COX-1 activity levels

Cultures were lysed and homogenised by freeze-thaw with dry ice and sonication in cold buffer. COX activity was evaluated using a COX Activity Assay Kit (760151, Cayman Chemical). Samples were prepared as indicated by manufacturer's instructions and readings were obtained with the Tecan i-control, software 1.10.4.0 (Tecan, Crailsheim, Germany).

\section{IL-6 ELISA procedure}

Supernatants from untreated and treated cell culture were assessed for IL-6 secretion by ELISA assay (DY206; R and D systems) according to the manufacturer's instruction. Basically, wells of 96-well microtitre plate were coated (overnight, room temperature) with $2 \mu \mathrm{g} / \mathrm{ml}$ of mouse anti-human IL-6 capture antibody in $50 \mu \mathrm{l}$ of PBS. The plate was then blocked with $1 \% \mathrm{w} / \mathrm{v}$ bovine serum albumin in PBS for $2 \mathrm{~h}$ at room temperature. Standards and samples were then loaded in duplicates and incubated for $2 \mathrm{~h}$ at room temperature, followed by detection antibody (biotinylated goat anti-human IL-6) for another $2 \mathrm{~h}$ at room temperature. Streptavidin HRP was added for $20 \mathrm{~min}$ at room temperature and the reaction was visualised by the addition of $50 \mu$ l of TMB substrate for $10-20 \mathrm{~min}$. The reaction was stopped with $25 \mu \mathrm{l}$ sulphuric acid $\left(\mathrm{H}_{2} \mathrm{SO}_{4}, 2 \mathrm{n}\right)$ and absorbance was measured at $450 \mathrm{~nm}$ with a reduction in $570 \mathrm{~nm}$ using Tecan Infinite M200 plate reader. The plate was washed three times with washing buffer (PBS with $0.05 \%(\mathrm{v} / \mathrm{v})$ Tween 20$)$ after each step. A standard curve was established using the absorbance gotten from a serial dilution of recombinant IL-6 $(18.8 \mathrm{pg} / \mathrm{ml}-2000 \mathrm{pg} / \mathrm{ml})$.
Absolute concentrations of the samples were calculated using Prism 7 (Graphpad Software Inc).

Oxidative activity assays

The relative oxidative activity of cultures was measured with the MTT assay (Roche 11465007001) and peroxidase assay. Cultures were maintained and treated for 72 as previously described, before being harvested by gentle agitation. For the MTT assay, cells were reseeded into a well of a 96-well plate and incubated with the reagents provided as indicated by manufacturer's instructions. In brief, measurements were obtained at $550 \mathrm{~nm}$ and $690 \mathrm{~nm}$ with an automatic microplate reader (Tecan Infinite M200), followed by analysis of the difference in absorbance levels with the Tecan i-control, software 1.10.4.0 (Tecan, Crailsheim, Germany). For the peroxidase assay, reactions were prepared as indicated by manufacturer's instructions (cat no. MAK092, SigmaAldrich Ltd., USA) and analysed at $570 \mathrm{~nm}$ with the microplate reader and software. Each sample was tested in duplicate with the average of eight readings per well recorded.

\section{HPLC analysis and purification}

The Atlantis Prep C18 reverse-phase column (Waters) was first equilibrated with $10 \%$ acetonitrile, $0.1 \%$ trifluoroacetic acid (TFA) in water at a flow rate of $5 \mathrm{ml} / \mathrm{min}$ for $15 \mathrm{~min}$. $100 \mu \mathrm{l}$ of sample was then loaded onto the column and the mobile phase was changed to $50 \%$ acetonitrile, $0.1 \%$ TFA in water under a linear gradient over a period of $55 \mathrm{~min}$ at a flow rate of $5 \mathrm{ml} / \mathrm{min}$. For the next $5 \mathrm{~min}$, a linear gradient to $100 \%$ acetonitrile containing $0.1 \%$ TFA was conducted. The eluted compounds were detected by measuring the absorbance at $270 \mathrm{~nm}$ and $475 \mathrm{~nm}$. Desired fractions were collected, frozen in liquid nitrogen and lyophilised using a VirTis benchtop freeze dryer.

\section{Mass spectrometry}

HPLC-MS analyses of the compounds were carried out on a 6550 iFunnel QTOF, connected with a 1290 UHPLC system (AGILENT, Singapore). The Zorbax Eclipse Plus C18, $2.1 \mathrm{~mm} \times 100 \mathrm{~mm}$ column, with $1.8 \mu \mathrm{m}$ particle size (AGILENT) was first equilibrated with $1 \%$ acetonitrile, $0.1 \%$ formic acid in water for $0.6 \mathrm{~min}$ at a flow rate of $500 \mu \mathrm{l} / \mathrm{min}$. The samples were dissolved in $100 \mu \mathrm{l} \mathrm{DMSO,}$ and $5 \mu \mathrm{l}$ was loaded for the analysis. Separation of the compounds was performed with a gradient of $1 \%$ acetonitrile, $0.1 \%$ formic acid in water to $99 \%$ acetonitrile, $0.1 \%$ formic acid in water for over $5 \mathrm{~min}$. In total, $99 \%$ acetonitrile, $0.1 \%$ formic acid in water was then maintained for another $1 \mathrm{~min}$, with the column temperature set at $40^{\circ} \mathrm{C}$ throughout the run. The mass spectrometer was set to an MS scan range from 50 to $1000 \mathrm{~m} / \mathrm{z}$ at 3 scans/sec. Data were recorded with Masshunter Acquisition B6.0 (AGILENT) and analysed with Masshunter Qualitative Analysis software version 6 (AGILENT).

After MS detection, CHNS elemental analyses were conducted by the Elemental \& Thermal Laboratory in the Department of Chemistry, National University of Singapore. Elemental analysis was done using Vario MICRO cube elemental analyser, Elementar Analysensysteme $\mathrm{GmbH}$, Hanau, Germany. NMR analyses were subsequently carried out by the Nuclear Magnetic Resonance Laboratory (School of Physical and Mathematical Sciences, Chemistry and Biological Chemistry department, Nanyang Technological University). ${ }^{1} \mathrm{H}$ NMR spectra were performed using Bruker Avance III 400 (400 MHz). Chemical shifts (ppm) were recorded with a residual solvent peak as an internal reference.

RNA extraction

RNA was extracted from cultures using the RNeasy Mini Kit (Qiagen, Germany). Cultures were lysed and homogenised with freeze-thaw and shear force cell disruption in buffer RLT as provided. Total RNA was eluted from the RNeasy Mini columns 
with $5 \mu \mathrm{l}$ of RNase-free water. The rest of the procedure was performed according to the manufacturer's protocol.

\section{Reverse transcription}

In total, $1 \mu$ l of $50 \mu \mathrm{M}$ oligo (dT) 20 primers (Life Technologies) and $1 \mu \mathrm{l}$ of $10 \mathrm{mM}$ dNTP Mix (Life Technologies) were added to $5 \mu \mathrm{l}$ of each lysed RNA sample. The sample was incubated at $65^{\circ} \mathrm{C}$ for $5 \mathrm{~min}$ and subsequently cooled on ice for at least $1 \mathrm{~min}$. In all, $1 \times$ first-strand buffer, 5 mM DTT, 40 U RNaseOUT Recombinant RNase Inhibitor, $200 \mathrm{U}$ SuperScript III RT (all Life Technologies) were added to a final volume of $20 \mu \mathrm{l}$. The following thermal setting was applied on a Verity 96-well Thermal Cycler (Applied Biosystems): $25^{\circ} \mathrm{C}$ for $5 \mathrm{~min}, 55^{\circ} \mathrm{C}$ for $60 \mathrm{~min}$ and $85^{\circ} \mathrm{C}$ for $5 \mathrm{~min}$. Each RT product was diluted to a final volume of $40 \mu \mathrm{l}$ to avoid qPCR inhibition.

\section{Real-time quantitative PCR (RT-qPCR)}

RT-qPCR was carried out in real-time using SYBR Green I detection chemistry on a Bio-Rad CFX96 Real-Time PCR Detection System (Bio-Rad Laboratories). Single-plex PCR was performed in a final volume of $10 \mu \mathrm{l}$, containing $300 \mathrm{nM}$ of each primer (Integrated DNA Technologies), $1 \times$ FastStart SYBR Green Master mix (Roche) and $1 \mu \mathrm{l}$ of diluted RT product. Gene primer pairs used in this study were selected from PrimerBank (https://pga.mgh.harvard. edu/primerbank/) and are listed in Table S4. In silico PCR was confirmed with UCSC genome browser (https://genome.ucsc.edu/) for each primer set validation. All primer specificities were confirmed with a single peak during melting curve analyses. The following thermal setting was applied on the RT-qPCR Cycler: $95^{\circ} \mathrm{C}$ for $10 \mathrm{~min}$, followed by 40 cycles of amplification $\left(95^{\circ} \mathrm{C}\right.$ for $20 \mathrm{sec}, 60^{\circ} \mathrm{C}$ for $30 \mathrm{sec}$ and $72{ }^{\circ} \mathrm{C}$ for $20 \mathrm{sec}$ ) and a final additional incubation at $72{ }^{\circ} \mathrm{C}$ for $7 \mathrm{~min}$. Gene expression data were normalised to two housekeeping genes (GADPH and $(\mathrm{BBB})$ with the following equation: relative expression $=$ $2^{-(C q(g e n e}$ of interest)-mean Cq(housekeeping genes)) . All measurements were performed in duplicate and mean values of relative expression are shown.

\section{Statistical analysis}

All error bars represented standard deviation of triplicate cultures from different samples. Groups were compared using the Student's $t$ test to evaluate associations between independent variables and the $p$ values were obtained using 0.01 or 0.05 significance levels, two-tailed. Adjusted multivariate analyses for continuous independent variables (to other variables) required larger sample sizes and were not utilised in this study. Resultant viability percentages were normalised to that obtained from samples in the last channel (lowest drug concentration). A fourparameter logistic equation was employed using Microsoft Excel ${ }^{\circledR}$ (Redmond, WA) for curve fitting analysis to determine $\mathrm{IC}_{50}$ values. The $I C_{50}$ value was obtained as the concentration value at which the curve passed through the $50 \%$ normalised response value corresponding to the percentage of cell death ( $y$ axis).

Availability of data and materials

The data sets supporting the conclusions of this article are stored in a secured research database and may be available upon presentation of formal approval.

\section{ACKNOWLEDGEMENTS}

We are grateful to the personnel who have provided technical support and facility usage located at the Singapore-MIT Alliance for Research and Technology (SMART) -BioSystems and Micromechanics (BioSyM) Laboratory, MBI and SIgN at A*STAR (Agency for Science Technology and Research). We thank Diego Pitta de Araujo of the MBI Science Communications Unit for the help in drawing 3D models of the device. We also appreciate the analysis and facility usage at the Department of Chemistry, NUS, for the elemental analysis. One or more authors have a pending patent related to this work. The experimental work and data collection were supported by the Singapore National Medical Research Council grant NMRC. This work was also supported by the Mechanobiology Institute and the Singapore-MIT Alliance for Research and Technology (SMART) BioSystems and Micromechanics (BioSyM) IRG, which are funded by the National Research Foundation, Prime Minister's Office, Singapore and by SIgN, which is funded by the Biomedical Research Council, A*STAR.

\section{AUTHOR CONTRIBUTIONS}

BLK, CTL and JH conceived and designed the experiments. BLK, GG, YPL, JF, WHY and SBL performed the experiments. BLK, YPL, JF, WHY, YSY and SLC analysed the data. GG, YPL, JF, SCW, YSY, SCL and CTL contributed reagents/materials/ analysis tools. BLK, GG, JSYL, YPL, JF, WHY, SLC, SCW, YSY, SCL, CTL and JH wrote the paper.

\section{ADDITIONAL INFORMATION}

Supplementary information is available for this paper at https://doi.org/10.1038/ s41416-018-0301-9.

Competing interests: The authors declare no competing interests.

Ethics approval and consent to participate: This study was approved by our institutional review board and local ethics committee (DSRB reference 2012/00105, 2012/00979, 2010/00270, and 2010/00691). Blood cells were obtained from healthy volunteers under the study approved by the institutional review board and local ethics committee (CIRB 2017/2806). All patients and volunteers gave their informed consent for inclusion in this study.

\section{REFERENCES}

1. Hughes, B. 2007 FDA drug approvals: a year of flux. Nat. Rev. Drug. Discov. 7 , 107-109 (2008).

2. Danson, S. et al. Phase I dose escalation and pharmacokinetic study of pluronic polymer-bound doxorubicin (SP1049C) in patients with advanced cancer. Br. J. Cancer 90, 2085-2091 (2004).

3. JLt, Hartman, Garvik, B. \& Hartwell, L. Principles for the buffering of genetic variation. Science 291, 1001-1004 (2001).

4. Kitano, H. A robustness-based approach to systems-oriented drug design. Nat. Rev. Drug. Discov. 6, 202-210 (2007).

5. McGranahan, N. \& Swanton, C. Cancer evolution constrained by the immune microenvironment. Cell 170, 825-827 (2017).

6. Singh, A. \& Settleman, J. EMT, cancer stem cells and drug resistance: an emerging axis of evil in the war on cancer. Oncogene 29, 4741-4751 (2010).

7. Hossain, M. A. et al. Aspirin enhances doxorubicin-induced apoptosis and reduces tumor growth in human hepatocellular carcinoma cells in vitro and in vivo. Int. J. Oncol. 40, 1636-1642 (2012).

8. Chen, W., Dong, J., Haiech, J., Kilhoffer, M. C. \& Zeniou, M. Cancer stem cell quiescence and plasticity as major challenges in cancer therapy. Stem Cells Int. 2016, 1740936 (2016).

9. Cole, S. P. et al. Overexpression of a transporter gene in a multidrug-resistant human lung cancer cell line. Science 258, 1650-1654 (1992).

10. Masciarelli, S. et al. Gain-of-function mutant p53 downregulates miR-223 contributing to chemoresistance of cultured tumor cells. Oncogene 33, 1601-1608 (2014).

11. Santos, J. C., Ribeiro, M. L., Sarian, L. O., Ortega, M. M. \& Derchain, S. F. Exosomesmediate microRNAs transfer in breast cancer chemoresistance regulation. Am. J. Cancer Res. 6, 2129-2139 (2016).

12. Batlle, E. \& Clevers, H. Cancer stem cells revisited. Nat. Med. 23, 1124-1134 (2017).

13. Danila, D. C., Pantel, K., Fleisher, M. \& Scher, H. I. Circulating tumors cells as biomarkers: progress toward biomarker qualification. Cancer J. 17, 438-450 (2011).

14. Slattum, G. M. \& Rosenblatt, J. Tumour cell invasion: an emerging role for basal epithelial cell extrusion. Nat. Rev. Cancer 14, 495-501 (2014).

15. Pattabiraman, D. R. \& Weinberg, R. A. Tackling the cancer stem cells - what challenges do they pose? Nat. Rev. Drug. Discov. 13, 497-512 (2014).

16. Meacham, C. E. \& Morrison, S. J. Tumour heterogeneity and cancer cell plasticity. Nature 501, 328-337 (2013).

17. Housman, G. et al. Drug resistance in cancer: an overview. Cancers (Basel). 6 , 1769-1792 (2014).

18. Bosetti, C., Rosato, V., Gallus, S., Cuzick, J. \& La Vecchia, C. Aspirin and cancer risk: a quantitative review to 2011. Ann. Oncol. 23, 1403-1415 (2012). 
19. Rothwell, P. M. et al. Long-term effect of aspirin on colorectal cancer incidence and mortality: 20-year follow-up of five randomised trials. Lancet 376, 1741-1750 (2010).

20. Crusz, S. M. \& Balkwill, F. R. Inflammation and cancer: advances and new agents. Nat. Rev. Clin. Oncol. 12, 584-596 (2015).

21. Elinav, E. et al. Inflammation-induced cancer: crosstalk between tumours, immune cells and microorganisms. Nat. Rev. Cancer 13, 759-771 (2013).

22. Flossmann, E. \& Rothwell, P. M. British Doctors Aspirin T, the UKTIAAT. Effect of aspirin on long-term risk of colorectal cancer: consistent evidence from randomised and observational studies. Lancet 369, 1603-1613 (2007).

23. Sandler, R. S. et al. A randomized trial of aspirin to prevent colorectal adenomas in patients with previous colorectal cancer. N. Eng. J. Med. 348, 883-890 (2003).

24. Funkhouser, E. M. \& Sharp, G. B. Aspirin and reduced risk of esophageal carcinoma. Cancer 76, 1116-1119 (1995).

25. Wang, W. H. et al. Non-steroidal anti-inflammatory drug use and the risk of gastric cancer: a systematic review and meta-analysis. J. Natl. Cancer Inst. 95, 1784-1791 (2003).

26. Rahman, M. A. et al. Sulindac and exisulind exhibit a significant antiproliferative effect and induce apoptosis in human hepatocellular carcinoma cell lines. Cancer Res. 60, 2085-2089 (2000).

27. Langman, M. J., Cheng, K. K., Gilman, E. A. \& Lancashire, R. J. Effect of antiinflammatory drugs on overall risk of common cancer: case-control study in general practice research database. BMJ 320, 1642-1646 (2000).

28. Khoo, B. L. et al. Liquid biopsy and therapeutic response: Circulating tumor cell cultures for evaluation of anticancer treatment. Sci. Adv. 2, e1600274 (2016).

29. Brighenti, E., Giannone, F. A., Fornari, F., Onofrillo, C. \& Govoni, M. \& Montanaro, L. Therapeutic dosages of aspirin counteract the IL- 6 induced pro-tumorigenic effects by slowing down the ribosome biogenesis rate. Oncotarget 7, 63226-63241 (2016)

30. Ogston, N. C. et al. Low-dose acetylsalicylic acid inhibits the secretion of interleukin-6 from white adipose tissue. Int. J. Obes. (Lond.). 32, 1807-1815 (2008).

31. Zhang, A., Dong, Z. \& Yang, T. Prostaglandin D2 inhibits TGF-beta1-induced epithelial-to-mesenchymal transition in MDCK cells. Am. J. Physiol. Ren. Physiol. 291, F1332-F1342 (2006).

32. Yoon, Y. S., Lee, Y. J., Choi, Y. H., Park, Y. M. \& Kang, J. L. Macrophages programmed by apoptotic cells inhibit epithelial-mesenchymal transition in lung alveolar epithelial cells via PGE2, PGD2, and HGF. Sci. Rep. 6, 20992 (2016).

33. Tian, J. et al. Cyclooxygenase- 2 regulates TGFbeta-induced cancer stemness in triple-negative breast cancer. Sci. Rep. 7, 40258 (2017).

34. Khoo, B. L. et al. Expansion of patient-derived circulating tumor cells from liquid biopsies using a CTC microfluidic culture device. Nat. Protoc. 13, 34-58 (2018).

35. Zheng, X., Cui, D., Xu, S., Brabant, G. \& Derwahl, M. Doxorubicin fails to eradicate cancer stem cells derived from anaplastic thyroid carcinoma cells: characterization of resistant cells. Int. J. Oncol. 37, 307-315 (2010)

36. Ryoo, I. G., Kim, G., Choi, B. H., Lee, S. H. \& Kwak, M. K. Involvement of NRF2 signaling in doxorubicin resistance of cancer stem cell-enriched colonospheres. Biomol. Ther. (Seoul.). 24, 482-488 (2016).

37. Khoo B. L., et al. Liquid biopsy and therapeutic response: circulating tumor cell cultures for evaluation of anticancer treatment. Sci. Adv. 2, e1600274 (2016).

38. Khoo, B. L. et al. Short-term expansion of breast circulating cancer cells predicts response to anti-cancer therapy. Oncotarget 6, 15578-15593 (2015).

39. Khoo B. L., Kumar P., Lim C., Thiery J. Genesis of Circulating Tumor Cells Through Epithelial-Mesenchymal Transition as a Mechanism for Distant Dissemination. in Circulating Tumor Cells: Ch 8, pp 139-182 (Springer, New York, NY 2016).

40. Pisco, A. O. \& Huang, S. Non-genetic cancer cell plasticity and therapy-induced stemness in tumour relapse: 'What does not kill me strengthens me'. Br. J. Cancer 112, 1725-1732 (2015).

41. Burrell, R. A., McGranahan, N., Bartek, J. \& Swanton, C. The causes and consequences of genetic heterogeneity in cancer evolution. Nature 501, 338-345 (2013).

42. Li, X. et al. Intrinsic resistance of tumorigenic breast cancer cells to chemotherapy. J. Natl. Cancer Inst. 100, 672-679 (2008).

43. Al-Hajj, M., Wicha, M. S., Benito-Hernandez, A., Morrison, S. J. \& Clarke, M. F. Prospective identification of tumorigenic breast cancer cells. Proc. Natl Acad. Sci. USA 100, 3983-3988 (2003).

44. Clevers, $\mathrm{H}$. The cancer stem cell: premises, promises and challenges. Nat. Med. 17 , 313-319 (2011).

45. Louie, E. et al. Identification of a stem-like cell population by exposing metastatic breast cancer cell lines to repetitive cycles of hypoxia and reoxygenation. Breast Cancer Res. 12, R94 (2010).

46. Hwang-Verslues, W. W. et al. Multiple lineages of human breast cancer stem/ progenitor cells identified by profiling with stem cell markers. PLOS. ONE 4, e8377 (2009).

47. Anderson, W. F., Chu, K. C., Chatterjee, N., Brawley, O. \& Brinton, L. A. Tumor variants by hormone receptor expression in white patients with node-negative breast cancer from the surveillance, epidemiology, and end results database. $J$. Clin. Oncol. 19, 18-27 (2001).

48. Botting, R. M. Inhibitors of cyclooxygenases: mechanisms, selectivity and uses. J. Physiol. Pharmacol. 57, 113-124 (2006).

49. Abramson, S. et al. Modes of action of aspirin-like drugs. Proc. Natl Acad. Sci. USA 82, 7227-7231 (1985).

50. Mohan, S. \& Epstein, J. B. Carcinogenesis and cyclooxygenase: the potential role of COX-2 inhibition in upper aerodigestive tract cancer. Oral. Oncol. 39, 537-546 (2003).

51. Liao, Z., Mason, K. A. \& Milas, L. Cyclo-oxygenase-2 and its inhibition in cancer: is there a role? Drugs 67, 821-845 (2007).

52. Hwang, D. H., Fung, V. \& Dannenberg, A. J. National Cancer Institute workshop on chemopreventive properties of nonsteroidal anti-inflammatory drugs: role of COX-dependent and -independent mechanisms. Neoplasia 4, 91-97 (2002).

53. Rigas, B. \& Kashfi, K. Cancer prevention: a new era beyond cyclooxygenase-2. J. Pharmacol. Exp. Ther. 314, 1-8 (2005).

54. $\mathrm{Xu}, \mathrm{L}$. et al. COX-2 inhibition potentiates antiangiogenic cancer therapy and prevents metastasis in preclinical models. Sci. Transl. Med. 6, 242 ra84 (2014).

55. Lamouille, S., Xu, J. \& Derynck, R. Molecular mechanisms of epithelialmesenchymal transition. Nat. Rev. Mol. Cell Biol. 15, 178-196 (2014).

56. Sansone, P. et al. Self-renewal of CD133(hi) cells by IL6/Notch3 signalling regulates endocrine resistance in metastatic breast cancer. Nat. Commun. 7, 10442 (2016).

57. Wang, X. et al. Sulforaphane improves chemotherapy efficacy by targeting cancer stem cell-like properties via the miR-124/IL-6R/STAT3 axis. Sci. Rep. 6, 36796 (2016).

58. Hunter, C. A. \& Jones, S. A. IL-6 as a keystone cytokine in health and disease. Nat. Immunol. 16, 448-457 (2015).

59. Mauer, J., Denson, J. L. \& Bruning, J. C. Versatile functions for IL-6 in metabolism and cancer. Trends Immunol. 36, 92-101 (2015).

60. Wunderlich, F. T. et al. Interleukin-6 signaling in liver-parenchymal cells suppresses hepatic inflammation and improves systemic insulin action. Cell Metab. 12, 237-249 (2010).

61. Gruber, S. et al. Obesity promotes liver carcinogenesis via Mcl-1 stabilization independent of IL-6Ralpha signaling. Cell Rep. 4, 669-680 (2013).

62. Ringborg, U. \& Platz, A. Chemotherapy resistance mechanisms. Acta Oncol. 35, 76-80 (1996).

63. Szakacs, G., Paterson, J. K., Ludwig, J. A., Booth-Genthe, C. \& Gottesman, M. M. Targeting multidrug resistance in cancer. Nat. Rev. Drug. Discov. 5, 219-234 (2006).

64. Uggeri, J. et al. Calcein AM is a detector of intracellular oxidative activity. Histochem. Cell Biol. 122, 499-505 (2004).

65. Imbeault, E. \& Gris, D. Assessment of oxidative metabolism. Methods Mol. Biol. 1031, 51-57 (2013).

66. Blanco, F. J., Guitian, R., Moreno, J., de Toro, F. J. \& Galdo, F. Effect of antiinflammatory drugs on COX-1 and COX-2 activity in human articular chondrocytes. J. Rheumatol. 26, 1366-1373 (1999).

67. Bellosillo, B. et al. Aspirin and salicylate induce apoptosis and activation of caspases in B-cell chronic lymphocytic leukemia cells. Blood 92, 1406-1414 (1998).

68. Rothwell, P. M. et al. Short-term effects of daily aspirin on cancer incidence, mortality, and non-vascular death: analysis of the time course of risks and benefits in 51 randomised controlled trials. Lancet 379, 1602-1612 (2012).

69. Peters, R. J. et al. Effects of aspirin dose when used alone or in combination with clopidogrel in patients with acute coronary syndromes: observations from the Clopidogrel in Unstable angina to prevent Recurrent Events (CURE) study. Circulation 108, 1682-1687 (2003).

70. Ruffin, M. Tt et al. Suppression of human colorectal mucosal prostaglandins: determining the lowest effective aspirin dose. J. Natl. Cancer Inst. 89, 1152-1160 (1997).

71. Mahmud, S., Franco, E. \& Aprikian, A. Prostate cancer and use of nonsteroidal antiinflammatory drugs: systematic review and meta-analysis. Br. J. Cancer 90, 93-99 (2004).

72. Khoo B. L., et al. Expansion of patient-derived circulating tumor cells from liquid biopsies using a CTC microfluidic culture device. Nat. Protoc. 13:34-58 (2017).

73. Shreders, A. et al. Prolonged benefit from ipilimumab correlates with improved outcomes from subsequent pembrolizumab. Cancer Immunol. Res. 4, 569-573 (2016).

74. Greenhough, A. et al. The COX-2/PGE2 pathway: key roles in the hallmarks of cancer and adaptation to the tumour microenvironment. Carcinogenesis $\mathbf{3 0}$, 377-386 (2009).

75. Antiplatelet Trialists' Collaboration. Collaborative overview of randomised trials of antiplatelet therapy--I: prevention of death. Myocardial infarction, and stroke by prolonged antiplatelet therapy in various categories of patients. BMJ 308, 81-106 (1994). 
Low-dose anti-inflammatory combinatorial therapy reduced cancer stem cell... BL Khoo et al.

76. Domingo, E. et al. Evaluation of PIK3CA mutation as a predictor of benefit from nonsteroidal anti-inflammatory drug therapy in colorectal cancer. J. Clin. Oncol. 31, 4297-4305 (2013).

77. Harris, R. E., Alshafie, G. A., Abou-Issa, H. \& Seibert, K. Chemoprevention of breast cancer in rats by celecoxib, a cyclooxygenase 2 inhibitor. Cancer Res. 60, 2101-2103 (2000).

78. Hu, P. J. et al. Chemoprevention of gastric cancer by celecoxib in rats. Gut 53, 195-200 (2004).

79. Han, J. A. et al. P53-mediated induction of Cox-2 counteracts p53- or genotoxic stress-induced apoptosis. EMBO J. 21, 5635-5644 (2002).

80. Taketo, M. M. Cyclooxygenase-2 inhibitors in tumorigenesis (part I). J. Natl. Cancer Inst. 90, 1529-1536 (1998).

81. Adhim, Z. et al. In vitro and in vivo inhibitory effect of three Cox-2 inhibitors and epithelial-to-mesenchymal transition in human bladder cancer cell lines. Br. J. Cancer 105, 393-402 (2011).

82. Steen, H. C. \& Gamero, A. M. The role of signal transducer and activator of transcription-2 in the interferon response. J. Interferon Cytokine Res. 32, 103-110 (2012).

83. Karaghiosoff, M. et al. Central role for type I interferons and Tyk2 in lipopolysaccharide-induced endotoxin shock. Nat. Immunol. 4, 471-477 (2003).

84. Thiery, J. P. Epithelial-mesenchymal transitions in tumour progression. Nat. Rev. Cancer 2, 442-454 (2002).

85. $\mathrm{Yu}, \mathrm{M}$. et al. Circulating breast tumor cells exhibit dynamic changes in epithelial and mesenchymal composition. Science 339, 580-584 (2013).

86. Spitzer, M. H. et al. Systemic immunity is required for effective cancer immunotherapy. Cell 168, 487-502 e15 (2017).

87. Nirschl, C. J. et al. IFNgamma-dependent tissue-immune homeostasis is co-opted in the tumor microenvironment. Cell 170, 127-141 e15 (2017).

88. Gupta, R. A. \& Dubois, R. N. Colorectal cancer prevention and treatment by inhibition of cyclooxygenase-2. Nat. Rev. Cancer 1, 11-21 (2001).

89. Huang, M. et al. Regulation of COX-2 expression and epithelial-to-mesenchymal transition by hypoxia-inducible factor-1alpha is associated with poor prognosis in hepatocellular carcinoma patients post TACE surgery. Int. J. Oncol. 48, 2144-2154 (2016).

90. Neil, J. R., Johnson, K. M., Nemenoff, R. A. \& Schiemann, W. P. Cox-2 inactivates Smad signaling and enhances EMT stimulated by TGF-beta through a PGE2dependent mechanisms. Carcinogenesis 29, 2227-2235 (2008).

91. Takai, E., Tsukimoto, M. \& Kojima, S. TGF-beta1 downregulates COX-2 expression leading to decrease of PGE2 production in human lung cancer A549 cells, which is involved in fibrotic response to TGF-beta1. PLoS. ONE 8, e76346 (2013).

92. Shureiqi, I. \& Lippman, S. M. Lipoxygenase modulation to reverse carcinogenesis. Cancer Res. 61, 6307-6312 (2001).

93. Lian Z.-J., Shih-Yu Hung, Ming-Ho Shen, Yang H. Rapid fabrication of semiellipsoid microlens using thermal reflow with two different photoresists. Microelectron. Eng. 115, 46-50 (2014).

94. Lee J.-H., Won-Seok Choi, Lee K.-H., Jun-Bo Yoon J. A simple and effective fabrication method for various 3D microstructures: backside 3D diffuser lithography J. Micromech. Microeng. 18, 12 (2008).

(i) Open Access This article is licensed under a Creative Commons Attribution 4.0 International License, which permits use, sharing, adaptation, distribution and reproduction in any medium or format, as long as you give appropriate credit to the original author(s) and the source, provide a link to the Creative Commons license, and indicate if changes were made. The images or other third party material in this article are included in the article's Creative Commons license, unless indicated otherwise in a credit line to the material. If material is not included in the article's Creative Commons license and your intended use is not permitted by statutory regulation or exceeds the permitted use, you will need to obtain permission directly from the copyright holder. To view a copy of this license, visit http://creativecommons. org/licenses/by/4.0/.

(c) The Author(s) 2019 